\title{
The computation of bounds for linear-functional outputs of weak solutions to the two-dimensional elasticity equations
}

\author{
N. Parés ${ }^{a}$ J. Bonet ${ }^{b}$ A. Huerta ${ }^{a}$ J. Peraire ${ }^{c} 1$ \\ ${ }^{a}$ Laboratori de Càlcul Numèric, Universitat Politècnica de Catalunya, Barcelona, \\ Spain \\ ${ }^{\mathrm{b}}$ Civil and Computational Engineering Centre, School of Engineering, University \\ of Wales, Swansea, UK \\ ${ }^{\mathrm{c}}$ Department of Aeronautics and Astronautics, Massachusetts Institute of \\ Technology, USA
}

\begin{abstract}
We present a method for the computation of upper and lower bounds for linear-functional outputs of the exact solutions to the two dimensional elasticity equations. The method can be regarded as a generalization of the well known complementary energy principle. The desired output is cast as the supremum of a quadratic-linear convex functional over an infinite dimensional domain. Using duality the computation of an upper bound for the output of interest is reduced to a feasibility problem for the complementary, or dual, problem. In order to make the problem tractable from a computational perspective two additional relaxations that preserve the bounding properties are introduced. First, the domain is triangulated and a domain decomposition strategy is used to generate a sequence of independent problems to be solved over each triangle. The Lagrange multipliers enforcing continuity are approximated using piecewise linear functions over the edges of the triangulation. Second, the solution of the adjoint problem is approximated over the triangulation using a standard Galerkin finite element approach. A lower bound for the output of interest is computed by repeating the process for the negative of the output. Reversing the sign of the computed upper bound for the negative of the output yields a lower bound for the actual output. The method can be easily generalized to three dimensions. However, a constructive proof for the

1 Corresponding author : J. Peraire, Department of Aeronautics and Astronuatics, Massachusetts Institute of Technology, 37-451, Cambridge, MA 02139, USA
\end{abstract}


existence of feasible solutions for the outputs of interest is only given in two dimensions. The computed bound gaps are found to converge optimally, that is, at the same rate as the finite element approximation. An attractive feature of the proposed approach is that it allows for a data set to be generated that can be used to certify and document the computed bounds. Using this data set and a simple algorithm, the correctness of the computed bounds can be established without recourse to the original code used to compute them. In the present paper, only computational domains whose boundary is made up of straight sided segments and polynomially varying loads are considered. Two examples are given to illustrate the proposed methodology.

\section{Introduction}

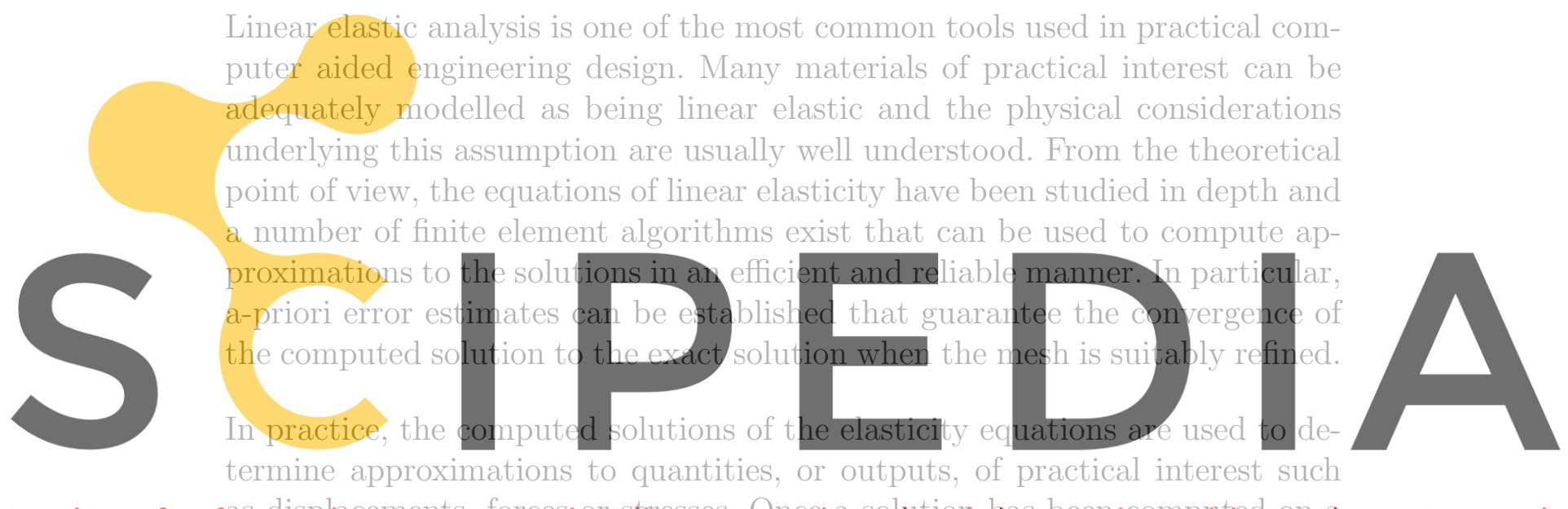

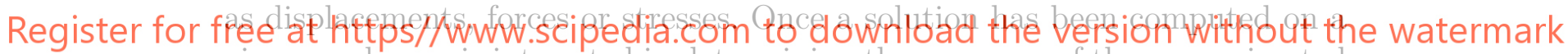
given mesh, one is interested in determining the accuracy of the approximated outputs. In order to address this question a number of a-posteriori error estimation methods have been proposed that attempt to quantify the error of the computed solution in either the energy norm $[1,3,5,9]$, or more relevantly, in functional outputs of practical interest such as displacements or stresses $[12,14,16]$. These a-posteriori approaches can be coupled with mesh adaptive strategies, e.g. [13], thus producing algorithms that, in principle, can be used to iterate from initial solution until a preset level of accuracy has been met.

Despite these numerous advances a fundamental issue still remains. Procedures that can be used to unambiguously certify the accuracy of the computed results have been elusive. The reasons for that are essentially twofold. Firstly, existing a-posteriori error estimation methods are considered to be quite reliable in practice but still involve uncertain ingredients. In some cases the expressions that bound the error involve continuity or interpolation constants which are non-computable and can approximated accurately only when the solution is well resolved $[14,16]$. In other situations, the bounds are uniform for 
any level of mesh refinement, but in practice are only computable by assuming that the exact solution can be locally represented on a conservatively refined mesh $[12,13]$. Alternatively, numerical integration may be required to evaluate integrals involving analytic functions [20]. Secondly, the above solution algorithms are implemented in computer codes which can easily have hundredths of thousands of lines of code, the correctness of which is virtually impossible to verify in practice.

In this paper we present a method to compute upper and lower bounds for linear outputs of interest of the exact weak solutions of the linear elasticity equations. The method is described in detail for two dimensions but the extension to the three dimensional case does not seem to present major difficulties. The approach presented can be interpreted as a generalization of the well known complementary energy principle [7]. This principle, which in its original form only yields bounds for the energy norm of the solution, has been known for many decades. Here, an extension to linear outputs of interest is presented.

The starting point for our bounds procedure are finite element approximations to the displacement solution and to the output dependent adjoint solution. These approximations are then post-processed to yield the so called

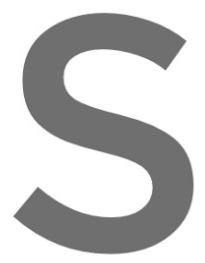
inter-element hy computation of
bounds is obtain
shown that the
the underlying cor brid fluxes.
locally
com by cate
oarse discr xes. The hybrid fluxes are the
quilibrated stross fields. The
alculating appropriate norms
d bounds are uniformly valid
cretization, but as expected, the
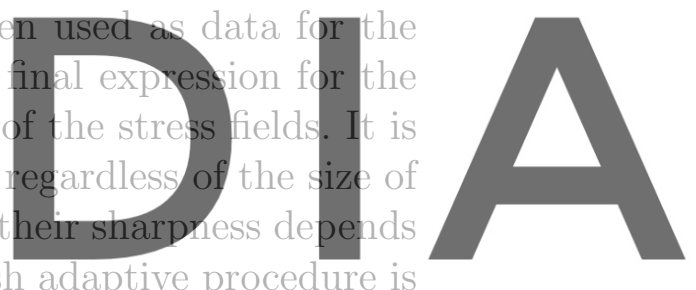

on the accuracy of the approximated solutions. A mesh adaptive procedure is

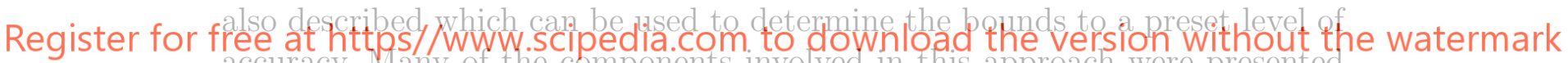
accuracy. Many of the components involved in this approach were presented in $[18,19]$ for scalar equations. In this paper we emphasize those aspects of the method which are particular to the elasticity equations.

An attractive feature of the proposed approach is that the piecewise polynomial equilibrated stress-like fields, which are computed as part of the bound process, can be used as certificates to guarantee the correctness of the computed bounds. It turns out that given a stress field it is easy to check whether this field corresponds to a valid certificate, and in the affirmative case it is straightforward to determine the value of the output that it can certify. In particular, the stress fields need to satisfy continuity of normal tractions across elements, and membership of an appropriate space.

The idea of a certificate that is computed simultaneously with the solution has many attractive features. In particular, a certificate consisting of the data set necessary to describe the piecewise polynomial stress-like fields could be used to document the computed results. We note that exercising the certificate 
does not require access to the code used to compute it and can be done with a simple algorithm which does not require solving any system of equations. A very important point is that, if a certificate meets all the necessary conditions, which in turn are easy to verify, then there is no need to certify the code used to compute it. In practice, the size of these certificates depends on the required level of certainty. As expected, we shall find that high levels of certainty, i.e. small bound gaps, will often require longer certificates (larger data sets) than those required to certify less sharp claims.

\section{Problem Definition}

The elasticity equations on a general two dimensional polygonal domain $\Omega$ are considered. The boundary, $\Gamma=\partial \Omega$, is divided into two complementary disjoint parts $\Gamma^{\mathrm{D}}$ and $\Gamma^{\mathrm{N}}$, where essential and Neumann boundary conditions are imposed, respectively. Furthermore, the boundary $\Gamma^{\mathrm{D}}$ is assumed to be a non empty set. The weak formulation of the problem consists of finding the displacements $\bar{u} \in \mathcal{U}$, such that
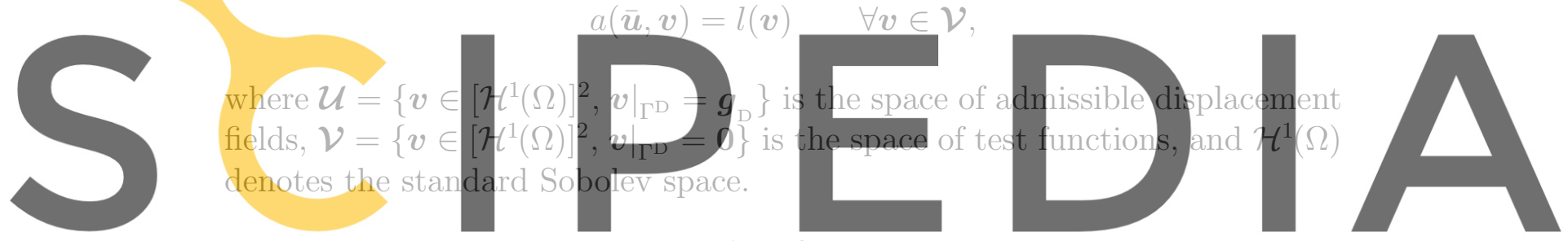

The linear forcing functional $l:\left[\mathcal{H}^{1}(\Omega)\right]^{2} \rightarrow \mathbb{R}$

Register for free at https//www.scipedia,com to download the version without the watermark

$$
l(v)=\int_{\Omega} f \cdot v \mathrm{~d} \Omega+\int_{\Gamma^{\mathrm{N}}} g \cdot v \mathrm{~d} \Gamma
$$

contains both the internal forces per unit volume $\boldsymbol{f} \in\left[\mathcal{H}^{-1}(\Omega)\right]^{2}$ an the Neumann boundary tractions $\boldsymbol{g} \in\left[\mathcal{H}^{-\frac{1}{2}}\left(\Gamma^{\mathrm{N}}\right)\right]^{2}$, and $a:\left[\mathcal{H}^{1}(\Omega)\right]^{2} \times\left[\mathcal{H}^{1}(\Omega)\right]^{2} \rightarrow \mathbb{R}$ is the symmetric positive definite bilinear form given by

$$
a(\boldsymbol{w}, \boldsymbol{v})=\int_{\Omega} \boldsymbol{\sigma}(\boldsymbol{w}): \boldsymbol{\varepsilon}(\boldsymbol{v}) \mathrm{d} \Omega
$$

Here, $\boldsymbol{\varepsilon}(\boldsymbol{v})$ is the second order deformation tensor, which is defined as the symmetric part of the gradient tensor $\nabla \boldsymbol{v}$, that is, $\boldsymbol{\varepsilon}(\boldsymbol{v})=\frac{1}{2}\left(\nabla \boldsymbol{v}+(\nabla \boldsymbol{v})^{\mathrm{T}}\right)$. The stress tensor $\boldsymbol{\sigma}(\boldsymbol{v})$, is related to the deformation tensor through a linear constitutive relation of the form, $\boldsymbol{\sigma}(\boldsymbol{v})=\mathbb{C}: \boldsymbol{\varepsilon}(\boldsymbol{v})$, where $\mathbb{C}$ is the fourthorder elasticity tensor. Throughout the paper the energy norm induced by the bilinear form $a(\cdot, \cdot)$ is denoted by $\|\cdot\|$, that is, $\|\boldsymbol{v}\|^{2}=a(\boldsymbol{v}, \boldsymbol{v})$.

Our goal is to compute bounds for output quantities of interest, $\bar{s}=\ell^{\mathcal{O}}(\overline{\boldsymbol{u}})$, 
where $\ell^{\mathcal{O}}:\left[\mathcal{H}^{1}(\Omega)\right]^{2} \rightarrow \mathcal{R}$ is a linear continuous functional defined as

$$
\ell^{\mathcal{O}}(\boldsymbol{v})=\int_{\Omega} \boldsymbol{f}^{\mathcal{O}} \cdot \boldsymbol{v} \mathrm{d} \Omega+\int_{\Gamma^{\mathrm{N}}} \boldsymbol{g}^{\mathcal{O}} \cdot \boldsymbol{v} \mathrm{d} \Gamma-a\left(\boldsymbol{u}^{\mathcal{O}}, \boldsymbol{v}\right),
$$

for given $\boldsymbol{f}^{\mathcal{O}} \in\left[\mathcal{H}^{-1}(\Omega)\right]^{2}, \boldsymbol{g}^{\mathcal{O}} \in\left[\mathcal{H}^{-\frac{1}{2}}\left(\Gamma^{\mathrm{N}}\right)\right]^{2}$ and $\boldsymbol{u}^{\mathbb{O}} \in\left[\mathcal{H}^{-1}(\Omega)\right]^{2}$. Note that this form may easily incorporate, as particular cases, displacements or tractions integrated over arbitrary subdomains or boundary segments.

For any given $\boldsymbol{u}^{D} \in \mathcal{U}$ we can write $\overline{\boldsymbol{u}}=\boldsymbol{u}^{D}+\boldsymbol{u}$, where $\boldsymbol{u} \in \mathcal{V}$ is the solution of

$$
a(\boldsymbol{u}, \boldsymbol{v})=l(\boldsymbol{v})-a\left(\boldsymbol{u}^{D}, \boldsymbol{v}\right)=: \ell(\boldsymbol{v}) \quad \forall \boldsymbol{v} \in \mathcal{V},
$$

and therefore the output of interest can be rewritten as

$$
\bar{s}=\ell^{\mathcal{O}}\left(\boldsymbol{u}^{D}\right)+\ell^{\mathcal{O}}(\boldsymbol{u}) .
$$

Working with $u \in \mathcal{V}$ rather than $\bar{u}$ has the advantage of avoiding the notational complexity introduced by the non-homogeneous Dirichlet boundary conditions. Thus, our goal will be to compute bounds for $s=\ell^{\mathcal{O}}(\boldsymbol{u})$, from which we can easily evaluate the bounds for $\bar{s}=\ell^{\mathcal{O}}(\bar{u})$.
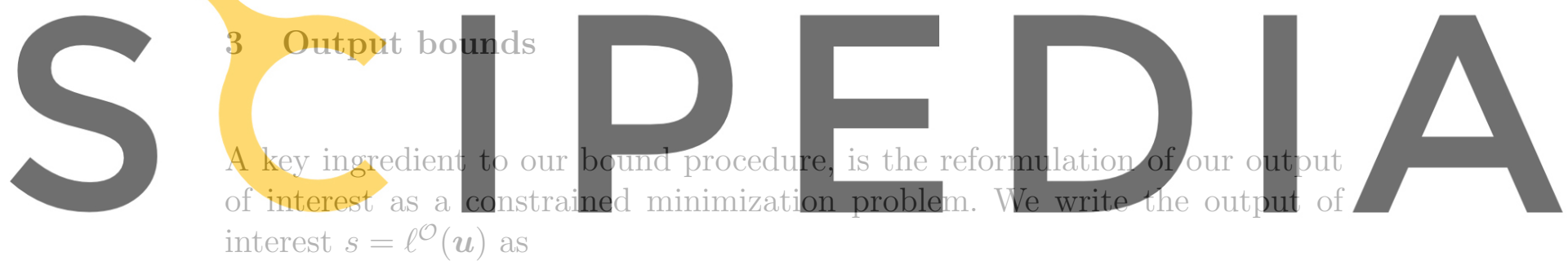

Register for free at https//www.scipedia.com to download the version without the watermark

$$
\begin{aligned}
s=\inf _{v \in \mathcal{V}} & \ell^{\mathcal{O}}(v)+\kappa^{2}(a(v, v)-\ell(v)) \\
\text { s.t. } & a(\boldsymbol{v}, \boldsymbol{\varphi})=\ell(\boldsymbol{\varphi}) \quad \forall \boldsymbol{\varphi} \in \mathcal{V},
\end{aligned}
$$

for all $\kappa \in \mathbb{R}$. The above statement can be easily verified by noting that, from (2), the constraint $a(\boldsymbol{v}, \boldsymbol{\varphi})=\ell(\boldsymbol{\varphi}), \forall \boldsymbol{\varphi} \in \mathcal{V}$ is only satisfied when $\boldsymbol{v}=\boldsymbol{u}$ and clearly $a(\boldsymbol{u}, \boldsymbol{u})-\ell(\boldsymbol{u})=0$. Now, the Lagrangian associated with the above constrained minimization problem problem is given by

$$
L(\boldsymbol{v}, \boldsymbol{\varphi})=\ell^{\mathcal{O}}(\boldsymbol{v})+\kappa^{2}(a(\boldsymbol{v}, \boldsymbol{v})-\ell(\boldsymbol{v}))+\ell(\boldsymbol{\varphi})-a(\boldsymbol{v}, \boldsymbol{\varphi}),
$$

and problem (3) becomes

$$
s=\inf _{\boldsymbol{v} \in \mathcal{V}} \sup _{\boldsymbol{\varphi} \in \mathcal{V}} L(\boldsymbol{v}, \boldsymbol{\varphi})
$$

A lower bound, $s^{-}$, for the output, $s$, can be easily found using the strong duality of convex minimization and the saddle point property of the Lagrange 
multipliers as

$$
s=\inf _{\boldsymbol{v} \in \mathcal{V}} \sup _{\boldsymbol{\varphi} \in \mathcal{V}} L(\boldsymbol{v}, \boldsymbol{\varphi})=\sup _{\boldsymbol{\varphi} \in \mathcal{V}} \inf _{\boldsymbol{v} \in \mathcal{V}} L(\boldsymbol{v}, \boldsymbol{\varphi}) \geq \inf _{\boldsymbol{v} \in \mathcal{V}} L(\boldsymbol{v}, \tilde{\boldsymbol{\varphi}}) \equiv s^{-} \quad \forall \tilde{\boldsymbol{\varphi}} \in \mathcal{V}
$$

where in order to obtain sharp bounds, it is important to use a good approximation $\tilde{\varphi}$ of the Lagrange multiplier. Thus, we see from (6), that the problem of computing a lower bound for the output of interest is cast as an unconstrained minimization problem.

The optimal value of for the Lagrange multiplier is obtained by solving the saddle problem (5) and is given by $\boldsymbol{\varphi}^{*}=\kappa^{2} \boldsymbol{u}+\boldsymbol{\psi}$ where $\boldsymbol{\psi} \in \mathcal{V}$ is the solution of the problem,

$$
a(\boldsymbol{v}, \boldsymbol{\psi})=\ell^{\mathcal{O}}(\boldsymbol{v}) \quad \forall \boldsymbol{v} \in \mathcal{V}
$$

called dual or adjoint problem with respect to the selected output $\ell^{\mathcal{O}}(\cdot)$. Note that due to the symmetry of $a(\cdot, \cdot)$, the only difference between the primal problem (2) for $\boldsymbol{u}$, and the dual problem (7) for $\boldsymbol{\psi}$, is only in the forcing data $\left(\boldsymbol{f}^{\mathcal{O}}\right.$ instead of $\boldsymbol{f}, \boldsymbol{g}^{\mathcal{O}}$ instead of $\boldsymbol{g}$ and $\boldsymbol{u}^{\mathcal{O}}$ instead of $\left.\boldsymbol{u}^{D}\right)$.

The solutions of the primal and dual problems are approximated by $\boldsymbol{u}_{h}$ and $\psi_{h}$ respectively, which lie in a finite element interpolation space $\boldsymbol{V}_{h} \subset \mathcal{V}$,
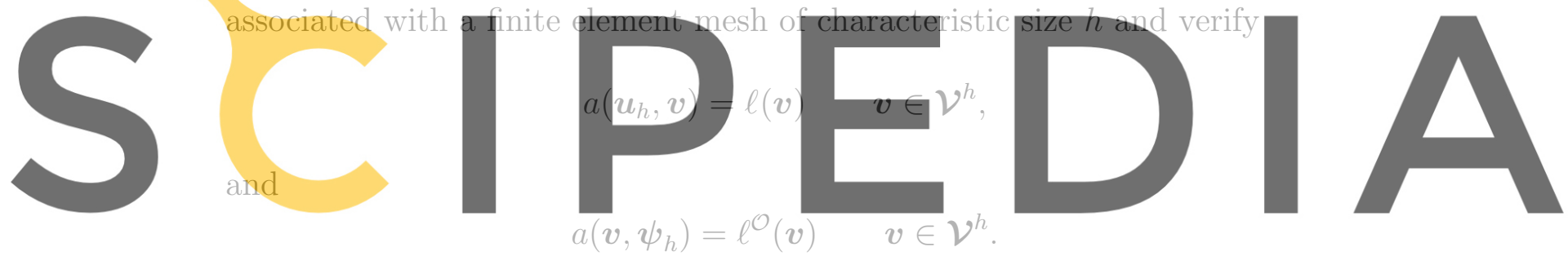

Register for free at https//www.scipedia.com to download the version without the watermark

An approximation to the Lagrange multiplier $\tilde{\varphi}$, is now obtained by setting

$\tilde{\varphi}=\kappa^{2} \boldsymbol{u}_{h}+\psi_{h}$. We note however that different options are also possible ( see for instance $[6,15])$. With our choice for $\tilde{\varphi}$ the optimization over $\boldsymbol{v}$ in (6), leads to

$$
s^{-}=\frac{1}{4}\left\|\kappa \boldsymbol{u}_{h}+\frac{1}{\kappa} \boldsymbol{\psi}_{h}\right\|^{2}-\frac{1}{4}\left\|\kappa \boldsymbol{u}-\frac{1}{\kappa} \boldsymbol{\psi}\right\|^{2}
$$

Remark 1 In the particular case when $\ell^{\mathcal{O}}(\cdot)=\ell(\cdot)$, then $s=\ell^{\mathcal{O}}(\boldsymbol{u})=\|\boldsymbol{u}\|^{2}$ and also $\boldsymbol{u}=\boldsymbol{\psi}$ and $\boldsymbol{u}_{h}=\boldsymbol{\psi}_{h}$. In this case, the lower bound we obtain for $\kappa=1$, is $s^{-}=\left\|\boldsymbol{u}_{h}\right\|^{2}$, which implies

$$
\left\|\boldsymbol{u}_{h}\right\|^{2} \leq\|\boldsymbol{u}\|^{2}
$$

This is the classical lower bound property of the energy norm of the finite element solution with respect to the exact solution norm.

An analogous expression for an upper bound, $s^{+}$, of $s$, is obtained by replacing 
$\ell^{\mathcal{O}}(\boldsymbol{v})$ by $-\ell^{\mathcal{O}}(\boldsymbol{v})$ in the original optimization problem (3) to obtain,

$$
\begin{gathered}
-s=\inf _{\boldsymbol{v} \in \mathcal{V}}-\ell^{\mathcal{O}}(\boldsymbol{v})+\kappa^{2}(a(\boldsymbol{v}, \boldsymbol{v})-\ell(\boldsymbol{v})) \\
\text { s.t. } \quad a(\boldsymbol{v}, \boldsymbol{\varphi})=\ell(\boldsymbol{\varphi}) \quad \forall \boldsymbol{\varphi} \in \mathcal{V} .
\end{gathered}
$$

The optimal multiplier in this case is approximated by $\tilde{\boldsymbol{\varphi}}=\kappa^{2} \boldsymbol{u}_{h}-\boldsymbol{\psi}_{h}$, and the optimization process yields

$$
-s^{+}=\frac{1}{4}\left\|\kappa \boldsymbol{u}_{h}-\frac{1}{\kappa} \boldsymbol{\psi}_{h}\right\|^{2}-\frac{1}{4}\left\|\kappa \boldsymbol{u}+\frac{1}{\kappa} \boldsymbol{\psi}\right\|^{2}
$$

which is equivalent to

$$
s^{+}=\frac{1}{4}\left\|\kappa \boldsymbol{u}+\frac{1}{\kappa} \boldsymbol{\psi}\right\|^{2}-\frac{1}{4}\left\|\kappa \boldsymbol{u}_{h}-\frac{1}{\kappa} \boldsymbol{\psi}_{h}\right\|^{2}
$$

\section{Remark 2 (Bounds for the output of the error) If the particular lift of} $\overline{\boldsymbol{u}}$ is its finite element approximation, that is $\boldsymbol{u}^{D}=\overline{\boldsymbol{u}}_{h}$, then $\boldsymbol{u}$ is the error in the finite element approximation, $\boldsymbol{u}=\overline{\boldsymbol{u}}-\overline{\boldsymbol{u}}_{h}=\boldsymbol{e}$, and $\boldsymbol{u}_{h}=0$. In this case, the previous methodology would lead after some algebra to bounds for $s=\ell^{\mathcal{O}}(e)$
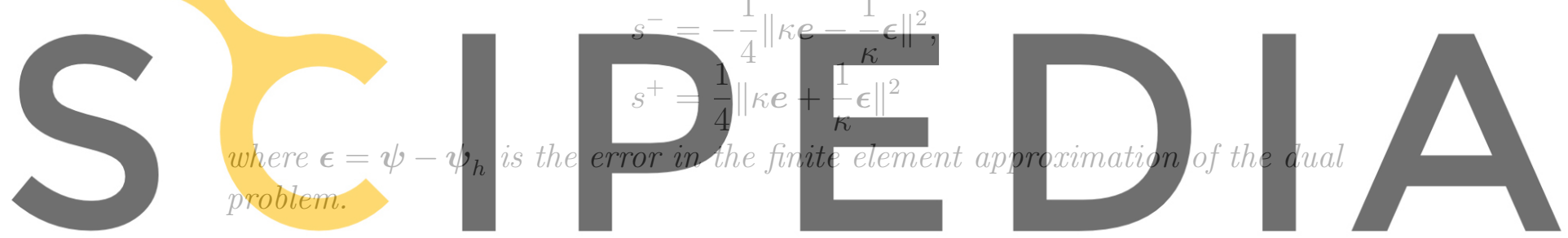

Writing together the expressions for the upper and lower bounds we have,

Register for free at https//www.scipedia.com to download the version without the watermark

$$
\frac{1}{4}\left\|\kappa u_{h}+\frac{1}{\kappa} \psi_{h}\right\|^{2}-\frac{1}{4}\left\|\kappa u-\frac{1}{\kappa} \psi\right\|^{2} \leq s \leq \frac{1}{4}\left\|\kappa u+\frac{1}{\kappa} \psi\right\|^{2}-\frac{1}{4}\left\|\kappa u_{h}-\frac{1}{\kappa} \psi_{h}\right\|^{2} .
$$

It is clear that these expressions are non-computable, since they depend on the exact solution of both the primal and dual problems. However, they illustrate our basic approach to obtaining bounds for outputs of interest: if we can compute upper bounds $\|\kappa \boldsymbol{u} \pm(1 / \kappa) \boldsymbol{\psi}\|_{\text {UB }}^{2}$ for $\|\kappa \boldsymbol{u} \pm(1 / \kappa) \boldsymbol{\psi}\|^{2}$, then, we can write computable expressions for the output bounds as

$$
\frac{1}{4}\left\|\kappa \boldsymbol{u}_{h}+\frac{1}{\kappa} \boldsymbol{\psi}_{h}\right\|^{2}-\frac{1}{4}\left\|\kappa \boldsymbol{u}-\frac{1}{\kappa} \boldsymbol{\psi}\right\|_{\mathrm{UB}}^{2} \leq s \leq \frac{1}{4}\left\|\kappa \boldsymbol{u}+\frac{1}{\kappa} \boldsymbol{\psi}\right\|_{\mathrm{UB}}^{2}-\frac{1}{4}\left\|\kappa \boldsymbol{u}_{h}-\frac{1}{\kappa} \boldsymbol{\psi}_{h}\right\|^{2} .
$$

In the next section, we present an approach for computing upper bounds for the energy norm of the solution of the elasticity equations. This result is then generalized in Section 5 to compute the upper bounds for the linear combination of the primal and dual functions, $\kappa \boldsymbol{u} \pm(1 / \kappa) \boldsymbol{\psi}$. 


\section{Upper bounds for the energy norm}

Consider the generalized elasticity problem with Neumann and homogeneous Dirichlet boundary conditions written in weak form as: find $\boldsymbol{z} \in \mathcal{V}$ such that

$$
a(\boldsymbol{z}, \boldsymbol{v})=\ell^{*}(\boldsymbol{v}) \quad \forall \boldsymbol{v} \in \mathcal{V}
$$

where

$$
\ell^{*}(\boldsymbol{v})=\int_{\Omega} \boldsymbol{f}^{*} \cdot \boldsymbol{v} \mathrm{d} \Omega+\int_{\Gamma^{\mathrm{N}}} \boldsymbol{g}^{*} \cdot \boldsymbol{v} \mathrm{d} \Gamma-a\left(\boldsymbol{u}^{*}, \boldsymbol{v}\right) .
$$

It is clear that any linear combination of the primal and dual solutions, $\alpha \boldsymbol{u}+$ $\beta \boldsymbol{\psi}, \alpha, \beta \in \mathbb{R}$, is the solution of problem (10) with $\boldsymbol{f}^{*}=\alpha \boldsymbol{f}+\beta \boldsymbol{f}^{\mathcal{O}}, \boldsymbol{g}^{*}=$ $\alpha \boldsymbol{g}+\beta \boldsymbol{g}^{\mathcal{O}}$ and $\boldsymbol{u}^{*}=\alpha \boldsymbol{u}^{D}+\beta \boldsymbol{u}^{\mathcal{O}}$. In particular, the choice $\alpha=\kappa, \beta= \pm 1 / \kappa$ will be used later to obtain the required upper bounds for $\|\kappa u \pm(1 / \kappa) \psi\|^{2}$.

In this section we consider the problem of computing an upper bound for $\|\boldsymbol{z}\|^{2}$. We recall that $\|\boldsymbol{z}\|^{2}$ can be obtained as the solution of the optimization procedure

$$
\|\boldsymbol{z}\|^{2}=\sup _{\boldsymbol{v} \in \mathcal{V}} 2 \ell^{*}(\boldsymbol{v})-a(\boldsymbol{v}, \boldsymbol{v})
$$
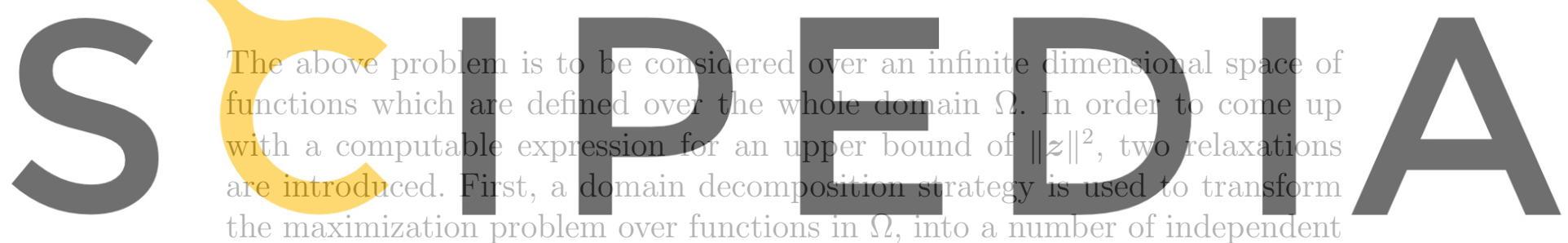

the maximization problem over functions in $\Omega$, into a number of independent

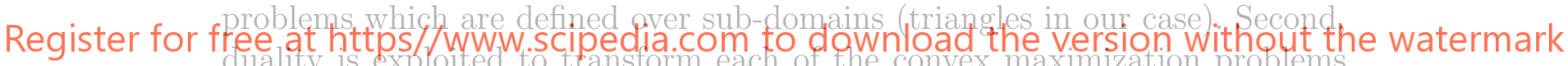

into a feasibility problem for the diral functional which is shown to yiold on

upper bound for the optimal solution.

\subsection{Domain decomposition}

We consider a triangulation of the computational domain $\Omega$ into $\mathrm{n}_{\mathrm{el}}$ triangles and denote by $\Omega_{k}$ a generic triangle, $k=1, \ldots \mathrm{n}_{\mathrm{el}}$. Let $\Gamma_{h}$ be the set of all the edges in the mesh, and $\boldsymbol{\Lambda}=\prod_{k=1}^{\mathrm{n}_{\mathrm{el}}}\left[\mathcal{H}^{-\frac{1}{2}}\left(\partial \Omega_{k}\right)\right]^{2}$ the space of integrable tractions in $\Gamma_{h}$. The set of all the interior edges of the mesh is denoted by $\Gamma^{\mathrm{I}}$, that is $\Gamma_{h}=\Gamma \cup \Gamma^{\mathrm{I}}$. For each edge $\gamma \in \Gamma_{h}$ a unit normal direction, $\boldsymbol{n}^{\gamma}$, is assigned such that, if $\gamma$ is an exterior edge, $\boldsymbol{n}^{\gamma}$ coincides with the outward unit normal to $\Gamma$. Similarly, given an element $\Omega_{k}$ and an edge of this element $\gamma \in \partial \Omega_{k}$, the outward normal to the element associated to $\gamma$ is denoted by $\boldsymbol{n}_{k}^{\gamma}$. Then, $\tau_{k}$ is 
defined as $\left.\tau_{k}\right|_{\gamma}=\boldsymbol{n}_{k}^{\gamma} \cdot \boldsymbol{n}^{\gamma}$, that is:

$$
\left.\tau_{k}\right|_{\gamma}=\boldsymbol{n}_{k}^{\gamma} \cdot \boldsymbol{n}^{\gamma}=\left\{\begin{array}{rll}
1 & \text { if } \boldsymbol{n}_{k}^{\gamma}=\boldsymbol{n}^{\gamma} \\
-1 & \text { if } \boldsymbol{n}_{k}^{\gamma}=-\boldsymbol{n}^{\gamma}
\end{array}\right.
$$

Note that if $\gamma=\partial \Omega_{k} \cap \partial \Omega_{l}$, then $\left.\tau_{k}\right|_{\gamma}+\left.\tau_{l}\right|_{\gamma}=0$.

The broken space $\widehat{\mathcal{V}}$ is introduced by relaxing in $\mathcal{V}$ both the Dirichlet homogeneous boundary conditions and the continuity of the functions across the edges of $\Gamma_{h}$, that is,

$$
\widehat{\mathcal{V}}=\left\{\hat{\boldsymbol{v}} \in\left[\mathcal{L}^{2}(\Omega)\right]^{2},\left.\hat{\boldsymbol{v}}\right|_{\Omega_{k}} \in\left[\mathcal{H}^{1}\left(\Omega_{k}\right)\right]^{2}, \forall \Omega_{k} \in \Omega\right\}
$$

Given a function in the broken space $\hat{v} \in \hat{\mathcal{V}}$, the jump of $\hat{\boldsymbol{v}}$ across the mesh edges is defined as

$$
\left.[\hat{v}]\right|_{\gamma}= \begin{cases}\left.\left.\hat{v}\right|_{\Omega_{k}} \tau_{k}\right|_{\gamma}+\left.\left.\hat{v}\right|_{\Omega_{l}} \tau_{l}\right|_{\gamma}, & \text { if } \gamma=\partial \Omega_{k} \cap \partial \Omega_{l} \in \Gamma^{\mathrm{I}} \\ \hat{v}, & \text { if } \gamma \in \Gamma,\end{cases}
$$
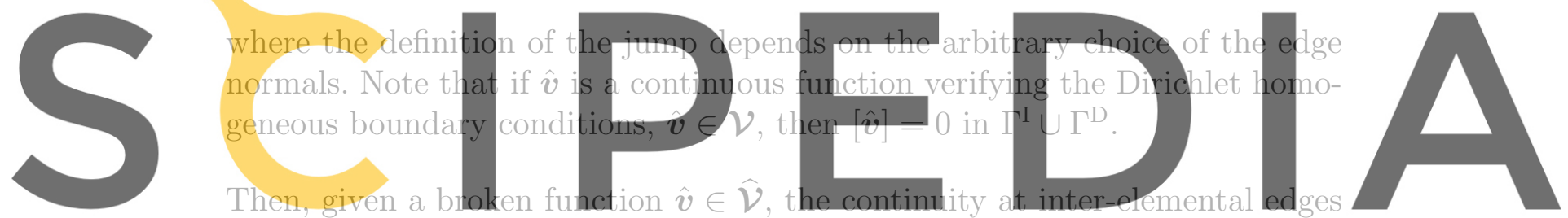

and Dirichlet homogeneous boundary conditions in $\Gamma^{\mathrm{D}}$ can be enforced weakly

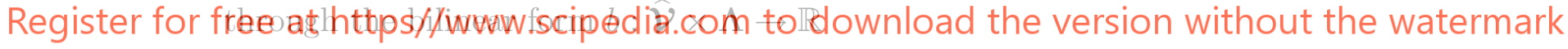

$$
b(\hat{v}, \boldsymbol{\lambda})=\sum_{\gamma \in \Gamma^{\mathrm{I}} \cup \Gamma^{\mathrm{D}}} \int_{\gamma} \boldsymbol{\lambda} \cdot[\hat{\boldsymbol{v}}] \mathrm{d} \Gamma=\left.\sum_{k=1}^{\mathrm{n}_{\mathrm{eI}}} \int_{\partial \Omega_{k} \backslash \Gamma^{\mathrm{N}}} \tau_{k} \boldsymbol{\lambda} \cdot \hat{\boldsymbol{v}}\right|_{\Omega_{k}} \mathrm{~d} \Gamma
$$

by imposing $b(\hat{\boldsymbol{v}}, \boldsymbol{\lambda})=0$ for all $\boldsymbol{\lambda} \in \boldsymbol{\Lambda}$. Therefore, the space of test functions $\mathcal{V}$ can be recovered as

$$
\mathcal{V}=\{\hat{\boldsymbol{v}} \in \hat{\mathcal{V}}, b(\hat{\boldsymbol{v}}, \boldsymbol{\lambda})=0, \forall \boldsymbol{\lambda} \in \boldsymbol{\Lambda}\}
$$

Let's denote by $\mathcal{V}_{k}$ the restriction of the broken space $\widehat{\mathcal{V}}$ to the element $\Omega_{k}$, that is, $\mathcal{V}_{k}=\left.\hat{\mathcal{V}}\right|_{\Omega_{k}}=\left[\mathcal{H}^{1}\left(\Omega_{k}\right)\right]^{2}$. Formally, any function $\boldsymbol{v}_{k} \in \mathcal{V}_{k}$ is not defined in the whole domain $\Omega$ but only in the element $\Omega_{k}$. In the following, any function $\boldsymbol{v}_{k} \in \mathcal{V}_{k}$ is naturally extended to $\Omega$ by setting the values outside $\Omega_{k}$ to zero. Then, a function $\hat{\boldsymbol{v}} \in \hat{\mathcal{V}}$ can be decomposed as the sum of its restrictions to each element $\boldsymbol{v}_{k}=\left.\hat{\boldsymbol{v}}\right|_{\Omega_{k}} \in \mathcal{V}_{k}$, that is, $\hat{\boldsymbol{v}}=\sum_{k=1}^{\mathrm{n}_{\mathrm{e}}} \boldsymbol{v}_{k}$, and $\hat{\mathcal{V}}=\bigoplus_{k=1}^{\mathrm{n}_{\mathrm{el}}} \mathcal{V}_{k}$. 
We can now rewrite the maximization problem of equation (11) as a constrained saddle problem defined over functions in $\hat{\mathcal{V}}$ and Lagrange multipliers in $\boldsymbol{\lambda}$ as,

$$
\|\boldsymbol{z}\|^{2}=\sup _{\hat{\boldsymbol{v}} \in \widehat{\mathcal{V}} \boldsymbol{i n f}} J(\hat{\boldsymbol{v}}, \boldsymbol{\lambda})
$$

where $J(\hat{\boldsymbol{v}}, \boldsymbol{\lambda})$ is the quadratic-linear Lagrangian which can be expressed using the local restrictions $\boldsymbol{v}_{k}$ of $\hat{\boldsymbol{v}}$ as

$$
J(\hat{\boldsymbol{v}}, \boldsymbol{\lambda})=\sum_{k=1}^{\mathrm{n}_{\mathrm{e} 1}} J_{k}\left(\boldsymbol{v}_{k}, \boldsymbol{\lambda}\right)
$$

where

$$
J_{k}\left(\boldsymbol{v}_{k}, \boldsymbol{\lambda}\right)=2 \ell_{k}^{*}\left(\boldsymbol{v}_{k}\right)-a_{k}\left(\boldsymbol{v}_{k}, \boldsymbol{v}_{k}\right)+2 b_{k}\left(\boldsymbol{v}_{k}, \boldsymbol{\lambda}\right) .
$$

Here, the subscript $k$ denotes the restriction of the linear and bilinear forms to the element $\Omega_{k}$, that is,

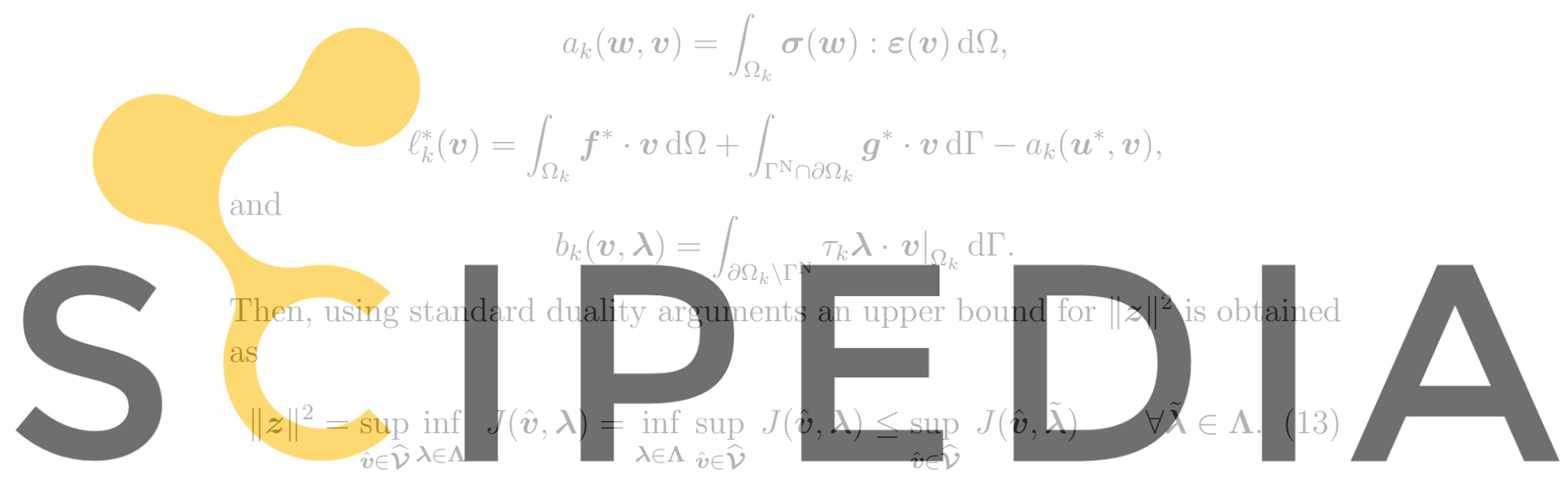

Register for free at https//www.scipedia.com to download the version without the watermark clearly, the Lagrange multiplier $\lambda$ has to be properly chosen in order to ensure

that the resulting maximization is bounded from above and that the resulting optimum is accurate. The importance of the weak imposition of the continuity requirement and the approximation of the Lagrange multiplier, is that once the Lagrange multiplier is fixed, the lagrangian $J(\hat{\boldsymbol{v}}, \tilde{\boldsymbol{\lambda}})$ decomposes into local elementary contributions, and the maximization in (13) decomposes into local maximization problems in the elements of the mesh.

In order to simplify the notation, we will rewrite $J_{k}(\cdot, \tilde{\boldsymbol{\lambda}})$ in a simpler way. We note that given a Lagrange multiplier $\tilde{\boldsymbol{\lambda}} \in \Gamma_{h}$, the values of $\tilde{\boldsymbol{\lambda}}$ in $\Gamma^{\mathrm{N}} \operatorname{do}$ not contribute to $J_{k}(\cdot, \tilde{\boldsymbol{\lambda}})$. Therefore we can define

$$
\left.\tilde{\boldsymbol{\lambda}}\right|_{\Gamma^{\mathrm{N}}}=\boldsymbol{g}^{*},
$$

so that,

$$
\int_{\Gamma^{\mathrm{N}} \cap \partial \Omega_{k}} \boldsymbol{g}^{*} \cdot \boldsymbol{v}_{k} \mathrm{~d} \Gamma+\int_{\partial \Omega_{k} \backslash \Gamma^{\mathrm{N}}} \tau_{k} \tilde{\boldsymbol{\lambda}} \cdot \boldsymbol{v}_{k} \mathrm{~d} \Gamma=\int_{\partial \Omega_{k}} \tau_{k} \tilde{\boldsymbol{\lambda}} \cdot \boldsymbol{v}_{k} \mathrm{~d} \Gamma,
$$


and therefore,

$$
J_{k}\left(\boldsymbol{v}_{k}, \tilde{\boldsymbol{\lambda}}\right)=2 \int_{\Omega_{k}} \boldsymbol{f}^{*} \cdot \boldsymbol{v}_{k} \mathrm{~d} \Omega+2 \int_{\partial \Omega_{k}} \tau_{k} \tilde{\boldsymbol{\lambda}} \cdot \boldsymbol{v}_{k} \mathrm{~d} \Gamma-2 a_{k}\left(\boldsymbol{u}^{*}, \boldsymbol{v}_{k}\right)-a_{k}\left(\boldsymbol{v}_{k}, \boldsymbol{v}_{k}\right) .
$$

Thus the global maximization of equation (13) can be decomposed as,

$$
\sup _{\hat{\boldsymbol{v}} \in \hat{\mathcal{V}}} J(\hat{\boldsymbol{v}}, \tilde{\boldsymbol{\lambda}})=\sum_{k=1}^{\mathrm{n}_{\mathrm{e}} \mathrm{I}} \sup _{\boldsymbol{v}_{k} \in \mathcal{V}_{k}} J_{k}\left(\boldsymbol{v}_{k}, \tilde{\boldsymbol{\lambda}}\right),
$$

allowing to obtain an upper bound for $\|\boldsymbol{z}\|^{2}$, maximizing the the local functionals $J_{k}(\cdot, \tilde{\boldsymbol{\lambda}})$ in each element of the mesh independently.

This local maximization problems, although local, can not be solved exactly because $\mathcal{V}_{k}$ is an infinite dimensional space. Moreover, if we replace $\mathcal{V}_{k}$ with a finite dimensional subspace, the upper bound property is lost.

\subsection{Complementary energy relaxation}

We consider now the problem of finding computable upper bounds for the local maximization problems (16), that is, find $\nu_{k} \in \mathbb{R}$ such that,

$$
\sup _{\boldsymbol{v}_{k} \in \mathcal{V}_{k}} J_{k}\left(\boldsymbol{v}_{k}, \tilde{\boldsymbol{\lambda}}\right) \leq \nu_{k}
$$

so that a global upper bound for $\|\boldsymbol{z}\|^{2}$ will be recovered as

$$
\|\boldsymbol{z}\|^{2} \leq \sum_{k=1}^{\mathrm{n}_{\mathrm{e} 1}} \sup _{\boldsymbol{v}_{k} \in \mathcal{V}_{k}} J_{k}\left(\boldsymbol{v}_{k}, \tilde{\boldsymbol{\lambda}}\right) \leq \sum_{k=1}^{\mathrm{n}_{\mathrm{e} 1}} \nu_{k} .
$$

The upper bounds $\nu_{k}$ are computed using a standard duality argument which transforms the problem of finding the maximum over the infinite dimensional space $\mathcal{V}_{k}$ to a problem of finding a feasible solution in an appropriate finite dimensional space.

Let $\mathcal{S}_{k}$ denote the space of componentwise square-integrable stress fields in $\Omega_{k}$, that is, $\mathcal{S}_{k}$ contains all the second-order tensors with $\sigma_{i j} \in \mathcal{L}^{2}\left(\Omega_{k}\right), \forall i, j$. Then, $\mathcal{S}_{k}^{e q}$ denotes the subset of $\mathcal{S}_{k}$ which contains all the equilibrated stress fields with respect to $\boldsymbol{f}^{*}, \tilde{\boldsymbol{\lambda}}$ and $\boldsymbol{u}^{*}$, that is, $\boldsymbol{\sigma}_{k} \in \mathcal{S}_{k}^{e q}$ verifies

$$
\int_{\Omega_{k}} \boldsymbol{\sigma}_{k}: \boldsymbol{\varepsilon}\left(\boldsymbol{v}_{k}\right) \mathrm{d} \Omega=\int_{\Omega_{k}} \boldsymbol{f}^{*} \cdot \boldsymbol{v}_{k} \mathrm{~d} \Omega+\int_{\partial \Omega_{k}} \tau_{k} \tilde{\boldsymbol{\lambda}} \cdot \boldsymbol{v}_{k} \mathrm{~d} \Gamma-a_{k}\left(\boldsymbol{u}^{*}, \boldsymbol{v}_{k}\right) \quad \forall \boldsymbol{v}_{k} \in \mathcal{V}_{k} .
$$

The stress fields in $\mathcal{S}_{k}^{e q}$ are usually referred to as being statically admissible. In addition, we define the complementary energy of a stress field $\boldsymbol{\sigma}_{k} \in \mathcal{S}_{k}$, as 
the value given by the the functional $J_{k}^{c}: \mathcal{S}_{k} \longrightarrow \mathbb{R}$,

$$
J_{k}^{c}\left(\boldsymbol{\sigma}_{k}\right)=\int_{\Omega_{k}} \boldsymbol{\sigma}_{k}: \mathbb{C}^{-1}: \boldsymbol{\sigma}_{k} \mathrm{~d} \Omega
$$

Lemma 1 If $\tilde{\boldsymbol{\lambda}}$ is such that $J_{k}\left(\boldsymbol{v}_{k}, \tilde{\boldsymbol{\lambda}}\right)$ is bounded from above for all $\boldsymbol{v}_{k} \in \mathcal{V}_{k}$, then the following duality relation holds,

$$
\sup _{\boldsymbol{v}_{k} \in \mathcal{V}_{k}} J_{k}\left(\boldsymbol{v}_{k}, \tilde{\boldsymbol{\lambda}}\right)=\inf _{\boldsymbol{\sigma}_{k} \in \mathcal{S}_{k}^{e q}} J_{k}^{c}\left(\boldsymbol{\sigma}_{k}\right)
$$

PROOF. Let $\boldsymbol{\sigma}_{k} \in \mathcal{S}_{k}^{e q}$ and $\boldsymbol{v}_{k} \in \mathcal{V}_{k}$, then

$$
\begin{aligned}
& 0 \leq \int_{\Omega_{k}}\left(\boldsymbol{\sigma}_{k}-\boldsymbol{\sigma}\left(\boldsymbol{v}_{k}\right)\right): \mathbb{C}^{-1}:\left(\boldsymbol{\sigma}_{k}-\boldsymbol{\sigma}\left(\boldsymbol{v}_{k}\right)\right) \mathrm{d} \Omega
\end{aligned}
$$

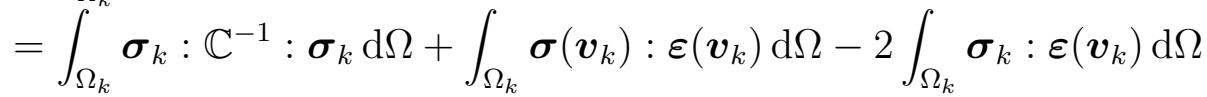

$$
\begin{aligned}
& =J_{k}^{c}\left(\boldsymbol{\sigma}_{k}\right)+a_{k}\left(\boldsymbol{v}_{k}, \boldsymbol{v}_{k}\right)-2 \int_{\Omega_{k}} \boldsymbol{\sigma}_{k}: \boldsymbol{\varepsilon}\left(\boldsymbol{v}_{k}\right) \mathrm{d} \Omega \text {. }
\end{aligned}
$$

Now, since $\boldsymbol{\sigma}_{k} \in \mathcal{S}_{k}^{e q}$ equation (18) holds true and

$$
2 \int_{\Omega_{k}} \boldsymbol{\sigma}_{k}: \boldsymbol{\varepsilon}\left(\boldsymbol{v}_{k}\right) \mathrm{d} \Omega-a_{k}\left(\boldsymbol{v}_{k}, \boldsymbol{v}_{k}\right)=J_{k}\left(\boldsymbol{v}_{k}, \tilde{\boldsymbol{\lambda}}\right),
$$

leading to

which implies $J_{k}\left(\boldsymbol{v}_{k}, \tilde{\boldsymbol{\lambda}}\right) \leq J_{k}^{c}\left(\boldsymbol{\sigma}_{k}\right)$.

$$
0 \leq J_{k}^{c}\left(\boldsymbol{\sigma}_{k}\right)-J_{k}\left(\boldsymbol{v}_{k}, \tilde{\boldsymbol{\lambda}}\right),
$$

Now, let $\overline{\boldsymbol{v}}_{k}$ be the point at which $J_{k}\left(\boldsymbol{v}_{k}, \tilde{\boldsymbol{\lambda}}\right)$ is maximum, that is,

$$
\left\|\overline{\boldsymbol{v}}_{k}\right\|_{k}^{2}=\sup _{\boldsymbol{v}_{k} \in \mathcal{V}_{k}} J_{k}\left(\boldsymbol{v}_{k}, \tilde{\boldsymbol{\lambda}}\right)
$$

where $\left\|\boldsymbol{v}_{k}\right\|_{k}^{2}=a_{k}\left(\boldsymbol{v}_{k}, \boldsymbol{v}_{k}\right)$. Moreover, the gradient condition for $\overline{\boldsymbol{v}}_{k}$ leads to

$$
a_{k}\left(\overline{\boldsymbol{v}}_{k}, \boldsymbol{v}_{k}\right)=\int_{\Omega_{k}} \boldsymbol{f}^{*} \cdot \boldsymbol{v}_{k} \mathrm{~d} \Omega+\int_{\partial \Omega_{k}} \tau_{k} \tilde{\boldsymbol{\lambda}} \cdot \boldsymbol{v}_{k} \mathrm{~d} \Gamma-a_{k}\left(\boldsymbol{u}^{*}, \boldsymbol{v}_{k}\right), \quad \forall \boldsymbol{v}_{k} \in \mathcal{V}_{k},
$$

from where it follows that $\overline{\boldsymbol{\sigma}}_{k}=\boldsymbol{\sigma}\left(\overline{\boldsymbol{v}}_{k}\right) \in \mathcal{S}_{k}^{e q}$.

Thus, $\forall \boldsymbol{\sigma}_{k} \in \mathcal{S}_{k}^{e q}, \forall \boldsymbol{v}_{k} \in \mathcal{V}_{k}$

$$
J_{k}\left(\boldsymbol{v}_{k}, \tilde{\boldsymbol{\lambda}}\right) \leq J_{k}\left(\overline{\boldsymbol{v}}_{k}, \tilde{\boldsymbol{\lambda}}\right)=\left\|\overline{\boldsymbol{v}}_{k}\right\|_{k}^{2}=J_{k}^{c}\left(\overline{\boldsymbol{\sigma}}_{k}\right) \leq J_{k}^{c}\left(\boldsymbol{\sigma}_{k}\right),
$$

and the lemma is proved.

Lemma 1 provides the key to the obtention of the local upper bounds $\nu_{k}$. It is sufficient to compute a statically admissible stress field in $\boldsymbol{\sigma}_{k}^{*} \in \mathcal{S}_{k}^{e q}$, and then evaluate its complementary energy, 


$$
\sup _{\boldsymbol{v}_{k} \in \mathcal{V}_{k}} J_{k}\left(\boldsymbol{v}_{k}, \tilde{\boldsymbol{\lambda}}\right)=\inf _{\boldsymbol{\sigma}_{k} \in \mathcal{S}_{k}^{e q}} J_{k}^{c}\left(\boldsymbol{\sigma}_{k}\right) \leq J_{k}^{c}\left(\boldsymbol{\sigma}_{k}^{*}\right)=\nu_{k}
$$

The remainder of the section is devoted to show that one can chose the statically admissible stress field to be piecewise polynomial provided that the forcing data $\boldsymbol{f}^{*}, \boldsymbol{g}^{*}$ and the displacement fields $\boldsymbol{u}^{*}$ are piecewise polynomial functions.

\subsection{Upper Bound Computation}

In order to construct the statically admissible stress field required in equation (19), it is first necessary to evaluate the Lagrange multiplier $\tilde{\boldsymbol{\lambda}}$ satisfying the necessary constraints. We will then construct the stress fields $\boldsymbol{\sigma}_{k}^{*}$ inside the elements.

\subsubsection{Lagrange multiplier approximation}

In order to obtain a sharp upper bound, the choice of the Lagrange multiplier is critical. In particular, the maximization in equation (17) must be bounded from above.

From equation (15), we note that the Lagrange multiplier $\tilde{\boldsymbol{\lambda}}$ is precisely the Neumann boundary condition for the local problems. That is, the traction distribution applied on the boundary of each element. When integrated over each element these tractions must therefore be equilibrated so that the local problems are solvable. This is equivalent to saying that

$$
\ell_{k}^{*}(\boldsymbol{v})+b_{k}(\boldsymbol{v}, \tilde{\boldsymbol{\lambda}})=0 \quad \forall \boldsymbol{v} \in \boldsymbol{P}_{\mathrm{sm}},
$$

where $\boldsymbol{P}_{\text {sm }}$, is the space of solid motions which includes any combination of translations and rotation. Moreover, since the optimal traction distribution is given by the tractions of the exact solution $\boldsymbol{z}$ over the edges of the elements, the Lagrange multipliers have to be both equilibrated and a good approximation to the tractions of the exact solution.

There are several known choices for the Lagrange multipliers which are approximations to the continuous tractions of the exact solution $\boldsymbol{z}$ at the inter element boundaries. Here we follow the the strategy proposed by Ladeveze et al. [9].

The approximated Lagrange multiplier is denoted by $\boldsymbol{\lambda}_{h}$ and it is a linear 
function in each edge of the mesh verifying

$$
b\left(\hat{\boldsymbol{v}}, \boldsymbol{\lambda}_{h}\right)=a\left(\boldsymbol{z}_{h}, \hat{\boldsymbol{v}}\right)-\ell^{*}(\hat{\boldsymbol{v}}) \quad \forall \hat{\boldsymbol{v}} \in \hat{\mathcal{V}}^{h}
$$

where $\boldsymbol{z}_{h}$ is the standard Galerkin finite element approximation of $\boldsymbol{z}$. We note that for any continuous $\hat{\boldsymbol{v}}, b\left(\hat{\boldsymbol{v}}, \boldsymbol{\lambda}_{h}\right)=0$, and therefore $a\left(\boldsymbol{z}_{h}, \hat{\boldsymbol{v}}\right)-\ell^{*}(\hat{\boldsymbol{v}})=0$ thus highlighting that $\boldsymbol{z}_{h}$ is indeed the finite element approximation to $\boldsymbol{z}$.

The above equations do not determine the Lagrange multiplier $\boldsymbol{\lambda}_{h}$ on $\Gamma^{\mathrm{N}}$. Therefore, $\boldsymbol{\lambda}_{h}$ is extended into $\Gamma^{\mathrm{N}}$ using equation (14).

Lemma 2 If $\boldsymbol{\lambda}_{h}$ verifies the equilibration conditions given in equation (21), then the local problems

$$
\sup _{\boldsymbol{v}_{k} \in \mathcal{V}_{k}} J_{k}\left(\boldsymbol{v}_{k}, \boldsymbol{\lambda}_{h}\right)
$$

are bounded from above.

PROOF. The null space of the bilinear form $a_{k}(\cdot, \cdot)$ is the three dimensional space of the rigid solid motions in the element $\Omega_{k}$ (translations and rotations), that is, $\boldsymbol{w}_{k}$ is a rigid solid body motion if and only if $a\left(\boldsymbol{v}_{k}, \boldsymbol{w}_{k}\right)=0, \forall \boldsymbol{v}_{k} \in \mathcal{V}_{k}$. Then, $J_{k}\left(\boldsymbol{w}_{k}, \boldsymbol{\lambda}_{h}\right)$ must vanish for any rigid motion $\boldsymbol{w}_{k}$, otherwise, given a rigid solid motion $\boldsymbol{w}_{k}$, for any $\alpha \in \mathbb{R}, \alpha \boldsymbol{w}_{k} \in \mathcal{V}_{k}$,

$$
J_{k}\left(\alpha \boldsymbol{w}_{k}, \boldsymbol{\lambda}_{h}\right)=2 \alpha\left(\ell_{k}^{*}\left(\boldsymbol{w}_{k}\right)+b_{k}\left(\boldsymbol{w}_{k}, \tilde{\boldsymbol{\lambda}}\right)\right)=\alpha J_{k}\left(\boldsymbol{w}_{k}, \boldsymbol{\lambda}_{h}\right)
$$

and this will lead to an unbounded maximization problem. Let's verify that $J_{k}\left(\boldsymbol{w}_{k}, \boldsymbol{\lambda}_{h}\right)=0$ for any $\boldsymbol{w}_{k}$ in the null space of $a_{k}(\cdot, \cdot)$.

Since the Lagrange multiplier $\boldsymbol{\lambda}_{h}$ is equilibrated, equation (21) is satisfied; thus, for any rigid solid motion $\boldsymbol{w}_{k}$ in the element $\Omega_{k}$,

$$
b_{k}\left(\boldsymbol{w}_{k}, \boldsymbol{\lambda}_{h}\right)=a_{k}\left(\boldsymbol{z}_{h}, \boldsymbol{w}_{k}\right)-\ell_{k}^{*}\left(\boldsymbol{w}_{k}\right)=-\ell_{k}^{*}\left(\boldsymbol{w}_{k}\right),
$$

since $\boldsymbol{w}_{k} \in \widehat{\mathcal{V}}^{h}$. Therefore,

$$
J_{k}\left(\boldsymbol{w}_{k}, \boldsymbol{\lambda}_{h}\right)=2 \ell_{k}^{*}\left(\boldsymbol{w}_{k}\right)+2 b_{k}\left(\boldsymbol{w}_{k}, \boldsymbol{\lambda}_{h}\right)-a_{k}\left(\boldsymbol{w}_{k}, \boldsymbol{w}_{k}\right)=0 .
$$

\subsubsection{Construction of an equilibrated stress field $\boldsymbol{\sigma}_{k} \in \mathcal{S}_{k}^{e q}$}

Once the Lagrange multipliers $\boldsymbol{\lambda}_{h}$ have been determined, an equilibrated stress field $\boldsymbol{\sigma}_{k} \in \mathcal{S}_{k}^{e q}$ has to be evaluated in order to obtain an upper bound for $\|\boldsymbol{z}\|^{2}$.

The existence of a piecewise polynomial stress field is established in the following theorem. 
Theorem 1 For any given forcing function $\left.\boldsymbol{f}^{*}\right|_{\Omega_{k}} \in\left[P_{r}\left(\Omega_{k}\right)\right]^{2}$ and any equilibrated Lagrange multiplier $\left.\boldsymbol{\lambda}_{h}\right|_{\partial \Omega_{k}} \in\left[P_{p}\left(\partial \Omega_{k}\right)\right]^{2}$, that is

$$
\int_{\Omega_{k}} \boldsymbol{f}^{*} \cdot \boldsymbol{v} \mathrm{d} \Omega+\int_{\partial \Omega_{k}} \tau_{k} \boldsymbol{\lambda}_{h} \cdot \boldsymbol{v} \mathrm{d} \Gamma=0 \quad \forall \boldsymbol{v} \in \boldsymbol{P}_{\mathrm{sm}}
$$

there exists at least one dual feasible solution $\boldsymbol{\sigma}_{k} \in \mathcal{S}_{k}=\left\{\boldsymbol{\sigma}, \sigma_{i j} \in \mathcal{L}^{2}\left(\Omega_{k}\right), \forall i, j\right\}$, verifying

$$
\int_{\Omega_{k}} \boldsymbol{\sigma}_{k}: \boldsymbol{\varepsilon}(\boldsymbol{v}) \mathrm{d} \Omega=\int_{\Omega_{k}} \boldsymbol{f}^{*} \cdot \boldsymbol{v} \mathrm{d} \Omega+\int_{\partial \Omega_{k}} \tau_{k} \boldsymbol{\lambda}_{h} \cdot \boldsymbol{v} \mathrm{d} \Gamma-a_{k}\left(\boldsymbol{u}^{*}, \boldsymbol{v}\right) \quad \forall \boldsymbol{v} \in \mathcal{V}_{k}
$$

which is piecewise polynomial of degree $q$ in each component, with $q \geq p$ and $q>r$.

A constructive proof of this theorem, based on some results presented in $[4,10]$, is given in the appendix.

Remark 3 For a linear equilibrated stress field (useful for linear applied tractions and constant force term), the local equilibrated stress fields are uniquely determined. Otherwise, $q>1$, there are extra degrees of freedom associated both to the internal boundaries and to the space of divergence free and trace free stress fields. This degrees of freedom are used to sharpen the bounds, that is, to minimize the value of $J_{k}^{c}\left(\boldsymbol{\sigma}_{k}\right)$.

The computation of the upper bound is summarized in the box shown in figure 1.

0.- Start with a piecewise polynomial representation of $\boldsymbol{u}^{D}$ and determine $\ell^{*}(\boldsymbol{v})$.

1.- Compute $\boldsymbol{z}_{h} \in \mathcal{V}^{h}$ s.t. $\quad a\left(\boldsymbol{z}_{h}, \boldsymbol{v}\right)=\ell^{*}(\boldsymbol{v}), \forall \boldsymbol{v} \in \mathcal{V}^{h}$.

2.- Compute $\boldsymbol{\lambda}_{h}$ s.t. $\quad b\left(\hat{\boldsymbol{v}}, \boldsymbol{\lambda}_{h}\right)=a\left(\boldsymbol{z}_{h}, \hat{\boldsymbol{v}}\right)-\ell^{*}(\hat{\boldsymbol{v}}), \forall \boldsymbol{v} \in \hat{\mathcal{V}}^{h}$, and impose $\left.\boldsymbol{\lambda}_{h}\right|_{\Gamma^{\mathrm{N}}}=\boldsymbol{g}^{*}$.

3.- For each element compute $\boldsymbol{\sigma}_{k} \in \mathcal{S}_{k}^{e q}$, that is, $\boldsymbol{\sigma}_{k} \in \mathcal{S}_{k}$ s.t.

$$
\int_{\Omega_{k}} \boldsymbol{\sigma}_{k}: \boldsymbol{\varepsilon}(\boldsymbol{v}) \mathrm{d} \Omega=\int_{\Omega_{k}} \boldsymbol{f}^{*} \cdot \boldsymbol{v} \mathrm{d} \Omega+\int_{\partial \Omega_{k}} \tau_{k} \boldsymbol{\lambda}_{h} \cdot \boldsymbol{v} \mathrm{d} \Gamma \quad \forall \boldsymbol{v} \in \mathcal{V}_{k},
$$

and compute $\nu_{k}=\int_{\Omega_{k}}\left(\boldsymbol{\sigma}_{k}-\boldsymbol{\sigma}\left(\boldsymbol{u}^{*}\right)\right): \mathbb{C}^{-1}:\left(\boldsymbol{\sigma}_{k}-\boldsymbol{\sigma}\left(\boldsymbol{u}^{*}\right)\right) \mathrm{d} \Omega$.

4.- Compute the upper bound for $\|\boldsymbol{z}\|^{2}$ as $\|\boldsymbol{z}\|^{2} \leq \sum_{k=1}^{\mathrm{n}_{\mathrm{el}}} \nu_{k}$.

Fig. 1. Upper bounds for the squared energy norm of the error 


\section{Bounds for the output of interest $s$}

We note that $\boldsymbol{z}^{ \pm}=\kappa \boldsymbol{u} \pm(1 / \kappa) \boldsymbol{\psi} \in \mathcal{V}$ is the solution of the boundary value problem,

$$
a\left(\boldsymbol{z}^{ \pm}, \boldsymbol{v}\right)=\kappa \ell(\boldsymbol{v}) \pm \frac{1}{\kappa} \ell^{\mathcal{O}}(\boldsymbol{v}) \quad \forall \boldsymbol{v} \in \mathcal{V}
$$

which is a particular case of $(10)$ with $\ell^{*}(\boldsymbol{v})=\kappa \ell(\boldsymbol{v}) \pm(1 / \kappa) \ell^{\mathcal{O}}(\boldsymbol{v})$.

Therefore, the approach described in the previous section we can used compute upper bounds for the energy norm of $\boldsymbol{z}^{ \pm}=\kappa \boldsymbol{u} \pm(1 / \kappa) \boldsymbol{\psi} \in \mathcal{V}$. These can then be used in expression (9) to yield computable expressions for the upper and lower bounds for $s$.

First, we compute approximations $\boldsymbol{u}_{h}, \boldsymbol{\psi}_{h} \in \mathcal{V}$ by solving,

$$
\begin{aligned}
& a\left(\boldsymbol{u}_{h}, \boldsymbol{v}\right)=\ell(\boldsymbol{v}) \quad \forall \boldsymbol{v} \in \mathcal{V}^{h} \\
& a\left(\boldsymbol{v}, \boldsymbol{\psi}_{h}\right)=\ell^{\mathcal{O}}(\boldsymbol{v})
\end{aligned}
$$

respectively, and set $\boldsymbol{z}_{h}^{ \pm}=\kappa \boldsymbol{u}_{h} \pm(1 / \kappa) \boldsymbol{\psi}_{h}$. Here, we assume that $\boldsymbol{u}^{D}$ and $\boldsymbol{u}^{\mathcal{O}}$ are piecewise polynomial over the elements of the working triangulation. For the particular case in which $\boldsymbol{u}^{D}$ and $\boldsymbol{u}^{\mathcal{O}}$ are the finite element approximations to $\boldsymbol{u}$ and $\boldsymbol{\psi}$, respectively, we will have $\boldsymbol{u}_{h}=\boldsymbol{\psi}_{h}=0$.

Second, using the strategy described in [9], compute Lagrange multipliers by equilibrating the primal and dual problems, namely, find $\boldsymbol{\lambda}_{h}^{\boldsymbol{u}}$ and $\boldsymbol{\lambda}_{h}^{\psi}$, such that,

$$
\begin{array}{lr}
b\left(\hat{\boldsymbol{v}}, \boldsymbol{\lambda}_{h}^{\boldsymbol{u}}\right)=a\left(\boldsymbol{u}_{h}, \hat{\boldsymbol{v}}\right)-\ell(\hat{\boldsymbol{v}}) & \forall \boldsymbol{v} \in \widehat{\mathcal{V}}^{h} \\
b\left(\hat{\boldsymbol{v}}, \boldsymbol{\lambda}_{h}^{\boldsymbol{\psi}}\right)=a\left(\hat{\boldsymbol{v}}, \boldsymbol{\psi}_{h}\right)-\ell^{\mathcal{O}}(\hat{\boldsymbol{v}}) & \forall \boldsymbol{v} \in \hat{\mathcal{V}}^{h} .
\end{array}
$$

Extend $\boldsymbol{\lambda}_{h}^{\boldsymbol{u}}$ and $\boldsymbol{\lambda}_{h}^{\boldsymbol{\psi}}$ at the Neumann boundaries according to $\left.\boldsymbol{\lambda}_{h}^{\boldsymbol{u}}\right|_{\Gamma^{\mathrm{N}}}=\boldsymbol{g}$ and $\left.\boldsymbol{\lambda}_{h}^{\psi}\right|_{\Gamma^{\mathrm{N}}}=\boldsymbol{g}^{\mathcal{O}}$, respectively. Finally, set $\boldsymbol{\lambda}_{h}^{ \pm}=\kappa \boldsymbol{\lambda}_{h}^{\boldsymbol{u}} \pm(1 / \kappa) \boldsymbol{\lambda}_{h}^{\psi}$.

Third, for each element in the mesh, we determine an equilibrated stress field $\boldsymbol{\sigma}_{k}^{ \pm}$verifying the equivalent of equation (23). That is, we compute $\boldsymbol{\sigma}_{k}^{\boldsymbol{u}}$ and $\boldsymbol{\sigma}_{k}^{\boldsymbol{\psi}}$ such that

$$
\begin{aligned}
& \int_{\Omega_{k}} \boldsymbol{\sigma}_{k}^{\boldsymbol{u}}: \boldsymbol{\varepsilon}(\boldsymbol{v}) \mathrm{d} \Omega=\int_{\Omega_{k}} \boldsymbol{f} \cdot \boldsymbol{v} \mathrm{d} \Omega+\int_{\partial \Omega_{k}} \tau_{k} \boldsymbol{\lambda}_{h}^{\boldsymbol{u}} \cdot \boldsymbol{v} \mathrm{d} \Gamma \quad \forall \boldsymbol{v} \in \mathcal{V}_{k},
\end{aligned}
$$

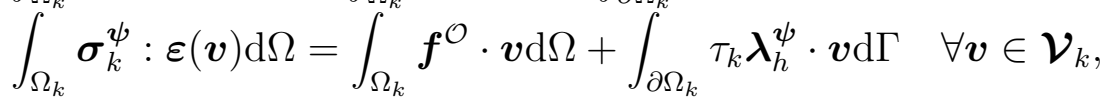

and set $\boldsymbol{\sigma}_{k}^{ \pm}=\kappa\left(\boldsymbol{\sigma}_{k}^{\boldsymbol{u}}-\boldsymbol{\sigma}_{k}^{D}\right) \pm(1 / \kappa)\left(\boldsymbol{\sigma}_{k}^{\boldsymbol{\psi}}-\boldsymbol{\sigma}_{k}^{\mathcal{O}}\right)$, where $\boldsymbol{\sigma}_{k}^{D}=\left.\boldsymbol{\sigma}\left(\boldsymbol{u}^{D}\right)\right|_{\Omega_{k}}$ and $\boldsymbol{\sigma}_{k}^{\mathcal{O}}=\left.\boldsymbol{\sigma}\left(\boldsymbol{u}^{\mathcal{O}}\right)\right|_{\Omega_{k}}$. 
Finally, the upper bound is found as

$$
\left\|\boldsymbol{z}^{ \pm}\right\| \leq \sum_{k=1}^{\mathrm{n}_{\mathrm{e} 1}} J_{k}^{c}\left(\boldsymbol{\sigma}_{k}^{ \pm}\right)=\kappa^{2} \nu^{\boldsymbol{u}}+\frac{1}{\kappa^{2}} \nu^{\boldsymbol{\psi}} \pm 2 \nu^{\boldsymbol{u} \boldsymbol{\psi}} \equiv\left\|\boldsymbol{z}^{ \pm}\right\|_{\mathrm{UB}}
$$

where

$$
\begin{aligned}
& \nu^{\boldsymbol{u}}=\sum_{k=1}^{\mathrm{n}_{\mathrm{e} 1}} J_{k}^{c}\left(\boldsymbol{\sigma}_{k}^{\boldsymbol{u}}-\boldsymbol{\sigma}_{k}^{D}\right), \\
& \nu^{\boldsymbol{\psi}}=\sum_{k=1}^{\mathrm{n}_{\mathrm{e} 1}} J_{k}^{c}\left(\boldsymbol{\sigma}_{k}^{\psi}-\boldsymbol{\sigma}_{k}^{\mathcal{O}}\right), \\
& \nu^{\boldsymbol{u} \psi}=\sum_{k=1}^{\mathrm{n}_{\mathrm{e} 1}} \int_{\Omega_{k}}\left(\boldsymbol{\sigma}_{k}^{\boldsymbol{u}}-\boldsymbol{\sigma}\left(\boldsymbol{u}^{D}\right)\right): \mathbb{C}^{-1}\left(\boldsymbol{\sigma}_{k}^{\psi}-\boldsymbol{\sigma}\left(\boldsymbol{u}^{\mathcal{O}}\right)\right) \mathrm{d} \Omega .
\end{aligned}
$$

Introducing expression (25) into (9) leads, after some algebra, to the following expressions for the upper and lower bounds of $s$,

$$
\begin{aligned}
& s^{+}=\frac{1}{2} s_{h}+\frac{1}{2} \nu^{\boldsymbol{u} \psi}+\frac{\kappa^{2}}{4}\left(\nu^{\boldsymbol{u}}-\left\|\boldsymbol{u}_{h}\right\|^{2}\right)+\frac{1}{4 \kappa^{2}}\left(\nu^{\boldsymbol{\psi}}-\left\|\boldsymbol{\psi}_{h}\right\|^{2}\right), \\
& s^{-}=\frac{1}{2} s_{h}+\frac{1}{2} \nu^{\boldsymbol{u} \boldsymbol{\psi}}-\frac{\kappa^{2}}{4}\left(\nu^{\boldsymbol{u}}-\left\|\boldsymbol{u}_{h}\right\|^{2}\right)-\frac{1}{4 \kappa^{2}}\left(\nu^{\boldsymbol{\psi}}-\left\|\boldsymbol{\psi}_{h}\right\|^{2}\right),
\end{aligned}
$$

where $s_{h}=\ell^{\mathcal{O}}\left(\boldsymbol{u}_{h}\right)$.

Following $[11,12]$, the bounds are optimized with respect to the arbitrary parameter $\kappa$. The optimal value is given by $\bar{\kappa}^{2}=\left(\sqrt{\nu^{\boldsymbol{\psi}}-\left\|\boldsymbol{\psi}_{h}\right\|^{2}}\right) /\left(\sqrt{\nu^{\boldsymbol{u}}-\left\|\boldsymbol{u}_{h}\right\|^{2}}\right)$. The resulting procedure to determine the bounds for $s$ is summarized in the box of figure 2 .

\section{Adaptive mesh refinement}

Once upper and lower bounds for the output quantity $s$ are computed, one can compute the bound average

$$
\underline{s}=\frac{1}{2}\left(s^{+}+s^{-}\right)=s_{h}+\nu^{u \psi}
$$

and the bound gap

$$
\Delta=s^{+}-s^{-}=\sqrt{\nu^{\boldsymbol{u}}-\left\|\boldsymbol{u}_{h}\right\|^{2}} \sqrt{\nu^{\boldsymbol{\psi}}-\left\|\boldsymbol{\psi}_{h}\right\|^{2}} .
$$

The bound average $\underline{s}$ is a new estimate of the output $s$, where its error with respect to $s$ can be easily bounded since

$$
|s-\underline{s}| \leq \frac{1}{2} \Delta .
$$


0.- Start from piecewise polynomial representations of $\boldsymbol{u}^{D}$ and $\boldsymbol{u}^{\mathcal{O}}$

1.- Compute $\boldsymbol{u}_{h} \in \mathcal{V}^{h}$ and $\boldsymbol{\psi}_{h} \in \mathcal{V}^{h}$ such that,

$$
\begin{aligned}
& a\left(\boldsymbol{u}_{h}, \boldsymbol{v}\right)=\ell(\boldsymbol{v}), \forall \boldsymbol{v} \in \mathcal{V}^{h} \\
& a\left(\boldsymbol{v}, \boldsymbol{\psi}_{h}\right)=\ell^{\mathcal{O}}(\boldsymbol{v}), \forall \boldsymbol{v} \in \mathcal{V}^{h}
\end{aligned}
$$

2.- Compute $\boldsymbol{\lambda}_{h}^{\boldsymbol{u}}$ and $\boldsymbol{\lambda}_{h}^{\boldsymbol{\psi}}$ such that,

$$
\begin{aligned}
& b\left(\hat{\boldsymbol{v}}, \boldsymbol{\lambda}_{h}^{\boldsymbol{u}}\right)=a\left(\boldsymbol{u}_{h}, \hat{\boldsymbol{v}}\right)-\ell(\hat{\boldsymbol{v}}), \forall \boldsymbol{v} \in \widehat{\mathcal{V}}^{h} \\
& b\left(\hat{\boldsymbol{v}}, \boldsymbol{\lambda}_{h}^{\psi}\right)=a\left(\hat{\boldsymbol{v}}, \boldsymbol{\psi}_{h}\right)-\ell^{\mathcal{O}}(\hat{\boldsymbol{v}}), \forall \boldsymbol{v} \in \hat{\mathcal{V}}^{h}
\end{aligned}
$$

and impose $\left.\boldsymbol{\lambda}_{h}^{\boldsymbol{u}}\right|_{\Gamma^{\mathrm{N}}}=\boldsymbol{g}$ and $\left.\boldsymbol{\lambda}_{h}^{\boldsymbol{\psi}}\right|_{\Gamma_{\mathrm{N}}}=\boldsymbol{g}^{\mathcal{O}}$.

3.- For each element find $\boldsymbol{\sigma}_{k}^{\boldsymbol{u}}, \boldsymbol{\sigma}_{k}^{\psi} \in \mathcal{\mathcal { S }}_{k}$ verifying

$$
\begin{aligned}
& \int_{\Omega_{k}} \boldsymbol{\sigma}_{k}^{\boldsymbol{u}}: \boldsymbol{\varepsilon}(\boldsymbol{v}) \mathrm{d} \Omega=\int_{\Omega_{k}} \boldsymbol{f} \cdot \boldsymbol{v} \mathrm{d} \Omega+\int_{\partial \Omega_{k}} \tau_{k} \boldsymbol{\lambda}_{h}^{\boldsymbol{u}} \cdot \boldsymbol{v} \mathrm{d} \Gamma, \forall \boldsymbol{v} \in \mathcal{V}_{k}, \\
& \int_{\Omega_{k}} \boldsymbol{\sigma}_{k}^{\psi}: \boldsymbol{\varepsilon}(\boldsymbol{v}) \mathrm{d} \Omega=\int_{\Omega_{k}} \boldsymbol{f}^{\mathcal{O}} \cdot \boldsymbol{v} \mathrm{d} \Omega+\int_{\partial \Omega_{k}} \tau_{k} \boldsymbol{\lambda}_{h}^{\psi} \cdot \boldsymbol{v} \mathrm{d} \Gamma, \forall \boldsymbol{v} \in \mathcal{V}_{k},
\end{aligned}
$$

and compute

$$
\begin{aligned}
& \nu_{k}^{\boldsymbol{u}}=\int_{\Omega_{k}}\left(\boldsymbol{\sigma}_{k}^{\boldsymbol{u}}-\boldsymbol{\sigma}\left(\boldsymbol{u}^{D}\right)\right): \mathbb{C}^{-1}:\left(\boldsymbol{\sigma}_{k}^{\boldsymbol{u}}-\boldsymbol{\sigma}\left(\boldsymbol{u}^{D}\right)\right) \mathrm{d} \Omega, \\
& \nu_{k}^{\psi}=\int_{\Omega_{k}}\left(\boldsymbol{\sigma}_{k}^{\psi}-\boldsymbol{\sigma}\left(\boldsymbol{u}^{\mathcal{O}}\right)\right): \mathbb{C}^{-1}:\left(\boldsymbol{\sigma}_{k}^{\psi}-\boldsymbol{\sigma}\left(\boldsymbol{u}^{\mathcal{O}}\right)\right) \mathrm{d} \Omega, \\
& \nu_{k}^{\boldsymbol{u} \psi}=\int_{\Omega_{k}}\left(\boldsymbol{\sigma}_{k}^{\boldsymbol{u}}-\boldsymbol{\sigma}\left(\boldsymbol{u}^{D}\right)\right): \mathbb{C}^{-1}:\left(\boldsymbol{\sigma}_{k}^{\psi}-\boldsymbol{\sigma}\left(\boldsymbol{u}^{\mathcal{O}}\right)\right) \mathrm{d} \Omega .
\end{aligned}
$$

4.- Compute the global quantities:

$$
\nu^{u}=\sum_{k=1}^{\mathrm{n}_{\mathrm{e} 1}} \nu_{k}^{u}, \quad \nu^{\psi}=\sum_{k=1}^{\mathrm{n}_{\mathrm{e} 1}} \nu_{k}^{\psi}, \quad \nu^{u \psi}=\sum_{k=1}^{\mathrm{n}_{\mathrm{e} 1}} \nu_{k}^{u \psi} .
$$

5.- Compute the bounds $s^{-}$and $s^{+}$for $s$ as

$$
\begin{aligned}
& s^{-}=\frac{1}{2} s_{h}+\frac{1}{2} \nu^{\boldsymbol{u} \boldsymbol{\psi}}-\frac{1}{2} \sqrt{\nu^{\boldsymbol{u}}-\left\|\boldsymbol{u}_{h}\right\|^{2}} \sqrt{\nu^{\boldsymbol{\psi}}-\left\|\boldsymbol{\psi}_{h}\right\|^{2}}, \\
& s^{+}=\frac{1}{2} s_{h}+\frac{1}{2} \nu^{\boldsymbol{u} \psi}+\frac{1}{2} \sqrt{\nu^{\boldsymbol{u}}-\left\|\boldsymbol{u}_{h}\right\|^{2}} \sqrt{\nu^{\boldsymbol{\psi}}-\left\|\boldsymbol{\psi}_{h}\right\|^{2}},
\end{aligned}
$$

where $s_{h}=\ell^{\mathcal{O}}\left(\boldsymbol{u}_{h}\right)$.

Fig. 2. Bounds for the output of interest $s$

If this error meets the desired requirements of accuracy the computation is concluded. On the other hand, if the level of precision does not meet the requirements a mesh adaptive procedure can be easily devised [13]. 
The bound gap can be written as,

$$
\Delta=\sum_{k=1}^{\mathrm{n}_{\mathrm{e} 1}} \frac{\bar{\kappa}^{2}}{2}\left(\nu_{k}^{\boldsymbol{u}}-\left\|\boldsymbol{u}_{h}\right\|_{k}^{2}\right)+\frac{1}{2 \bar{\kappa}^{2}}\left(\nu_{k}^{\boldsymbol{\psi}}-\left\|\boldsymbol{\psi}_{h}\right\|_{k}^{2}\right)=\sum_{k=1}^{\mathrm{n}_{\mathrm{e} 1}} \Delta_{k},
$$

where $\bar{\kappa}$ is the optimal value of the parameter $\kappa$ which optimizes the bounds. The above expression identifies the elemental contributions $\Delta_{k}$. These contributions can be shown to be always positive (since the complementary energy of an equilibrated stress field and the energy of an arbitrary displacement field, are upper and lower bounds to the energy of the exact solution, respectively) and can therefore be used as a local refinement indicator.

Then, given a target bound gap $\Delta_{\text {tol }}$, at each level of refinement, the elements with $\Delta_{k} \geq\left(\Delta_{\mathrm{tol}} / \mathrm{n}_{\mathrm{el}}\right)$ are refined. Numerical experimentation indicates that this strategy leads to a robust and reliable procedure to achieve the desired accuracy. The refined meshes are obtained using the mesh generator presented in $[17]$.

\section{$7 \quad$ Numerical Examples}

The presented method is illustrated with two numerical examples: a linearly forced square which has a regular solution for which an analytical expression exists, and a square plate with two interior rectangular cut-outs, the solution of which, has corner singularitites. The outputs of interest are in both cases displacements and reaction forces integrated over parts of the boundary. Linear finite elements approximations have been used for the adjoint and the hybrid fluxes have also been interpolated linearly over each edge. The equilibrated stress fields in the dual problem are also taken to be linearly varying in space.

The coarse mesh problems are solved using triangular linear finite elements, and the local equilibrated stress fields are taken to be piecewise linear in each triangle of the mesh. Four estimates of $s$ are considered: the upper and lower bounds ( $s^{+}$and $s^{-}$, respectively), their average, $\underline{s}=\left(s^{+}+s^{-}\right) / 2$, and also the output given by the finite element approximation, denoted by $s_{h}=\ell^{\mathcal{O}}\left(\boldsymbol{u}_{h}\right)$.

In the first example the analytical solution of the problem is known and the quality of the different estimates is measured with the following effectivity indices $\rho^{ \pm}=\left(s^{ \pm} / s\right)-1, \underline{\rho}=(\underline{s} / s)-1$, and $\rho_{h}=\left(s_{h} / s\right)-1$. Another measure of the accuracy of the bounds is given by the relative half bound gap,

$$
\rho_{\mathrm{G}}=\frac{1}{2} \frac{s^{+}-s^{-}}{|s|} \geq 0 \text {. }
$$

Since $s^{+}$and $s^{-}$are upper and lower bounds of $s$, the index $\rho_{\mathrm{G}}$ is an upper 
bound of the relative error between the approximation $\underline{s}$ and the exact output $s$, that is,

$$
\frac{|\underline{s}-s|}{|s|} \leq \rho_{\mathrm{G}} .
$$

In the second example, where the analytical solution is not known, the bound accuracy is measured in terms of the relative half bound gap, $\rho_{\mathrm{G}}$, which is re-defined as,

$$
\rho_{\mathrm{G}}=\frac{1}{2} \frac{s^{+}-s^{-}}{|\underline{s}|},
$$

where the exact output is replaced by the average estimate.

\subsection{Linearly forced square}

The plane stress elasticity equations are considered in the unity square $[0,1]^{2}$. On the left edge of the square, $x_{1}=0$, Dirichlet homogeneous boundary conditions are imposed in the $x_{2}$ direction, and in the left-lower corner, $(0,0)$, both the $x_{1}$ and $x_{2}$ displacements are prescribed to zero. Also, a linear normal traction, $\boldsymbol{g}=\left(x_{2}, 0\right)^{T}$, is applied at the right edge, $x_{1}=1$.

The analytical solution of the problem $\boldsymbol{u}=\left(u_{1}, u_{2}\right)$, is given by

$$
u_{1}\left(x_{1}, x_{2}\right)=\frac{1}{E} x_{1} x_{2}, \quad u_{2}\left(x_{1}, x_{2}\right)=-\frac{1}{2 E}\left(\nu x_{2}^{2}+x_{1}^{2}\right),
$$

where $E$ and $\nu$ are the Young's modulus and the Poisson's ratio.

The output considered is the weighted average normal displacement at the right edge,

$$
s=\int_{0}^{1} x_{2} u_{1}\left(1, x_{2}\right) d x_{2}=\frac{1}{3 E}
$$

It turns out that for this particular forcing and output, the primal and adjoint problems are the same. For this case, called compliance, the output is proportional to the energy norm of the solution and the finite element approximation directly provides a lower bound. The numerical results demonstrate that our method, while more expensive, leads to the same lower bound, doing no worse than the inherent bound of the finite element approximation.

Four uniform triangular meshes have been considered, the initial one with 18 elements $(h=1 / 3)$. The other meshes are obtained by uniformly subdividing each element of the previous mesh into 4 new elements. The results are summarized in the table 1 .

Figure 3 displays the results graphically and also illustrates the convergence rate of the bounds. The results for both the upper and lower bounds, average, 
Table 1

Bounds and effectivity indices in a series of uniformly refined meshes

\begin{tabular}{|c|c|ccc|ccc|c|}
\hline$h$ & $s_{h}$ & $s^{-}$ & $s^{+}$ & $\underline{s}$ & $\rho^{-}$ & $\rho^{+}$ & $\underline{\rho}$ & $\rho_{\mathrm{G}}$ \\
\hline $1 / 3$ & .3124 & .3124 & .5621 & .4372 & -.062740 & .686210 & .311720 & .3745 \\
\hline $1 / 6$ & .3264 & .3264 & .4370 & .3817 & -.020710 & .310880 & .145070 & .1658 \\
\hline $1 / 12$ & .3314 & .3314 & .3653 & .3484 & -.005710 & .095990 & .045140 & .0508 \\
\hline $1 / 24$ & .3328 & .3328 & .3419 & .3374 & -.001480 & .025640 & .012080 & .0136 \\
\hline $1 / 48$ & .3332 & .3332 & .3355 & .3344 & -.000370 & .006530 & .003080 & .0034 \\
\hline
\end{tabular}
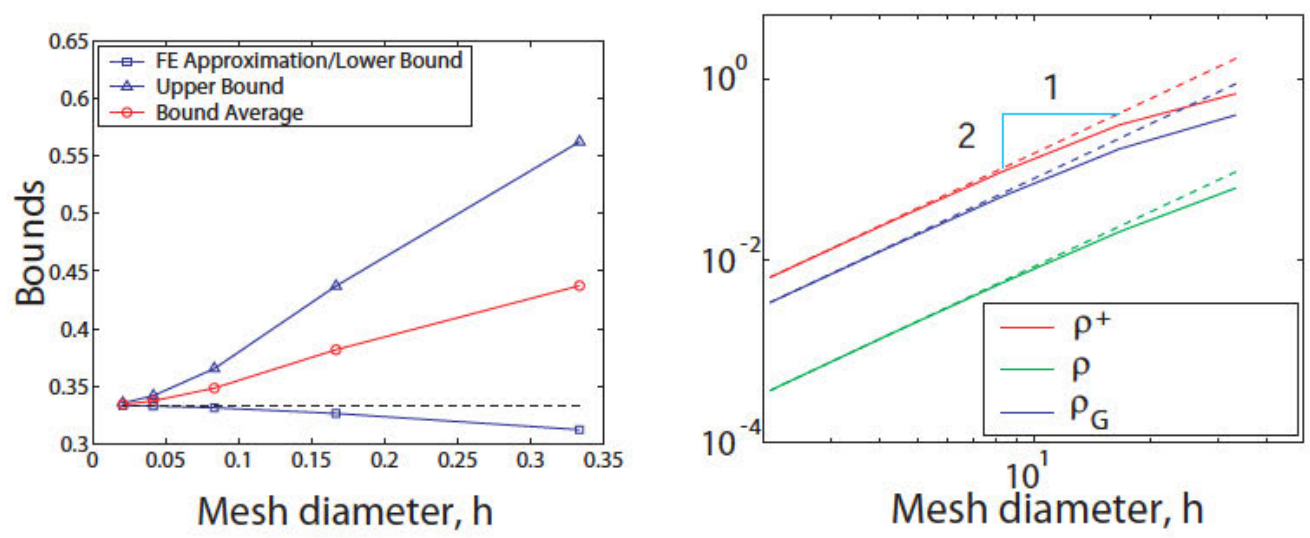

Fig. 3. Computed bounds for a a uniform $h$-refinement process (left) and its convergence (right)

and relative half bound gap, $\rho_{\mathrm{G}}$, asymptotically approach the finite element convergence rate of $\mathcal{O}\left(h^{2}\right)$.

\subsection{Square plate}

A square thin plate with two rectangular holes is considered. Normal tractions are applied on the left and right sides of the plate [13]. Since the problem is symmetric, only one fourth of the plate is considered, as shown in figure 4 .

Two outputs of interest are considered: the average normal displacement over the boundary $\Gamma_{0}$, and the integrated normal component of the traction in $\Gamma_{1}$, that is,

$$
\ell_{0}^{\mathcal{O}}(\boldsymbol{v})=\int_{\Gamma_{0}} \boldsymbol{v} \cdot \boldsymbol{n} d \Gamma, \quad \ell_{1}^{\mathcal{O}}(\boldsymbol{v})=\int_{\Gamma_{1}} \boldsymbol{n} \cdot \boldsymbol{\sigma}(\boldsymbol{v}) \boldsymbol{n} d \Gamma .
$$

Remark 4 The first output is already in the form of equation (1) with $\boldsymbol{g}^{\mathcal{O}}=$ $\left.\boldsymbol{n}\right|_{\Gamma_{0}}$ and $\boldsymbol{g}^{\mathcal{O}}=\mathbf{0}$ elsewhere. The second output, on the other hand, does not have the same form. In order to transform this output into the form (1) considered here, we introduce a continuous function $\chi$ such that $\left.\chi\right|_{\Gamma_{1}}=1$ and is 


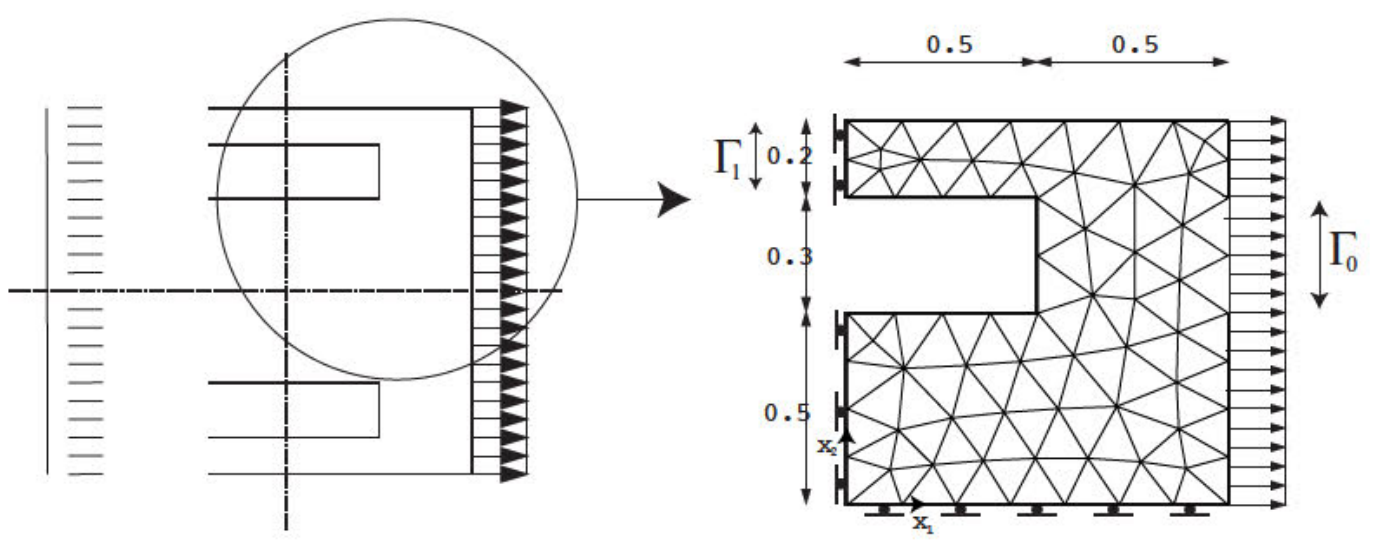

Fig. 4. Example 2: Model problem (left) and initial mesh (right)

equal to zero at all the other vertical boundaries. Then, if $\boldsymbol{n}^{1}=\left.\boldsymbol{n}\right|_{\Gamma_{1}}$, we have

$$
s=\ell_{1}^{\mathcal{O}}(\boldsymbol{u})=\int_{\Gamma_{1}} \boldsymbol{n} \cdot \boldsymbol{\sigma}(\boldsymbol{u}) \boldsymbol{n} d \Gamma=a\left(\boldsymbol{u}, \chi \boldsymbol{n}^{1}\right)=: \tilde{\ell_{1}^{\mathcal{O}}}(\boldsymbol{u}),
$$

and instead of working with the functional $\ell_{1}^{\mathcal{O}}(\cdot)$, we work with $\tilde{\ell_{1}^{\mathcal{O}}}(\cdot)$. This is much easier since this corresponds to $\boldsymbol{u}^{\mathcal{O}}=-\chi \boldsymbol{n}^{1}$ in equation (1).

Figure 5 and table 2 show the bounds obtained in this example. A nested sequence of meshes is considered. The initial mesh $\left(h_{\text {ini }}\right)$ is shown in figure 4 , and the refined meshes are obtained, as in the first example, dividing each element into 4 new ones. The function $\chi$ required in $\tilde{\ell_{1}^{\mathcal{O}}}(\cdot)$, is defined on the initial mesh by setting all the nodal values equal to zero except for those nodes on $\Gamma_{1}$ which are given a value of unity.

Unlike the first example, the outputs in (26) are general and the finite element approximation can no longer be guaranteed to provide a lower bound. This example shows that the bounds behave well even for problems with singularities. However, it is also observed that the convergence rate for the bounds, the finite element approximation $s_{h}$ and the bound average, is no longer $\mathcal{O}\left(h^{2}\right)$, although it is still faster than linear.

For the reaction output, $\ell \mathcal{O}_{1}(u)$, an adaptive procedure has been employed starting with the mesh shown in figure (4) where the bound gap $\Delta_{\text {ini }}$ is 0.1075 , and two target bound gaps have been considered $\Delta_{\text {tol }}=\frac{1}{2} \Delta_{\text {ini }}$ and $\Delta_{\text {tol }}=$ $\frac{1}{10} \Delta_{\text {ini }}$.

In order to achieve $\Delta_{\text {tol }}=\frac{1}{2} \Delta_{\text {ini }}$ four new meshes are generated, where the bound gap for the last mesh is $\Delta_{\mathrm{f}}=0.0471$. The resulting sequence of meshes can be seen in figure (6), where the local elementary contributions to the global bound gap are plotted in each element of the mesh. As can be seen not only the zone where the output is measured $\left(\Gamma_{1}\right)$ is refined, but also the corners where the solution is singular. 

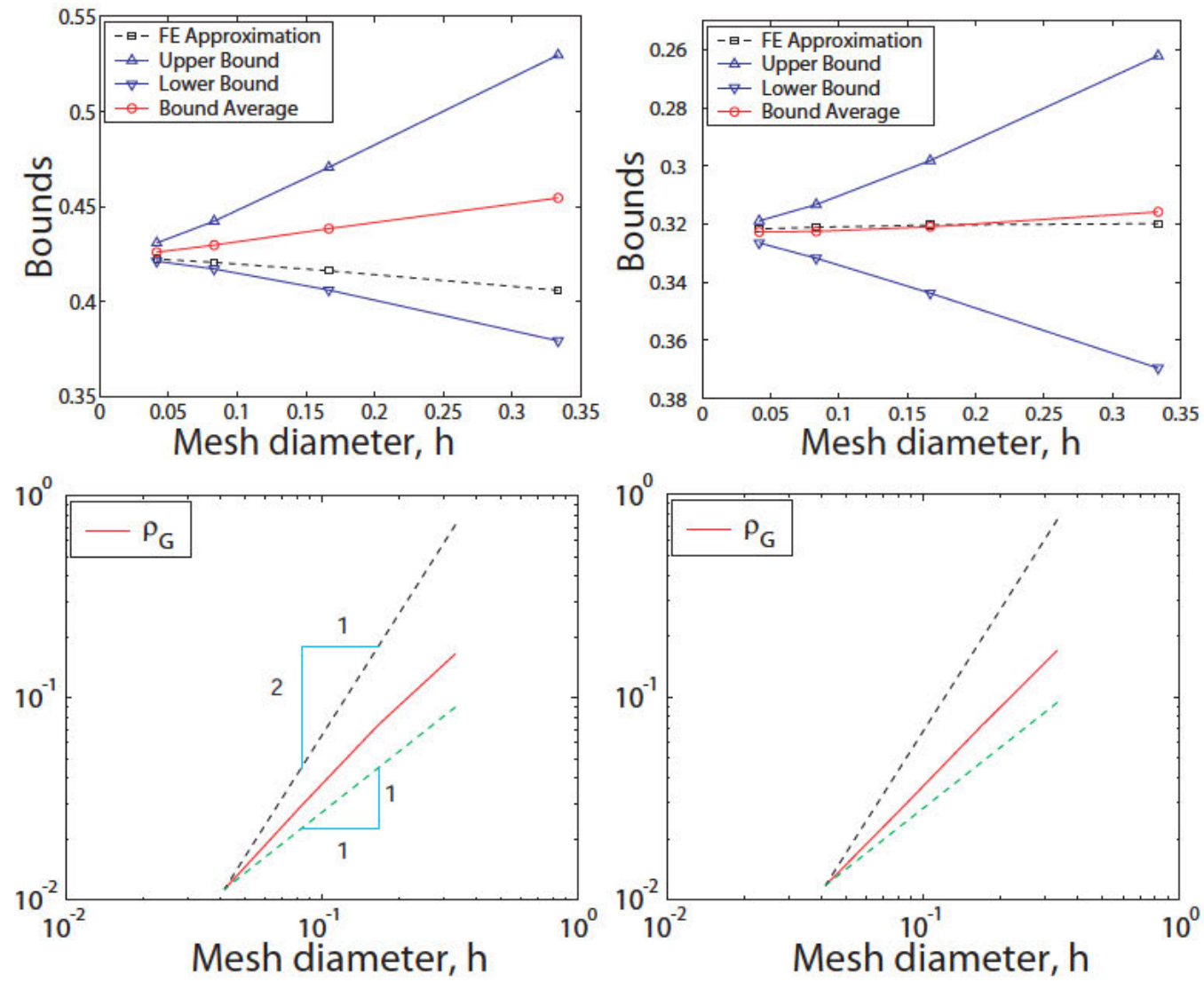

Fig. 5. Bounds convergence for a uniform $h$-refinement (up) and for the displacement output $\ell_{0}^{\mathcal{O}}(\boldsymbol{u})$ (left) and for the reaction output $\ell_{1}^{\mathcal{O}}(\boldsymbol{u})$ (right)

\begin{tabular}{|c|c|cccc|c|cccc|}
\hline & \multicolumn{5}{|c|}{ displacement average } & \multicolumn{5}{c|}{ reaction average } \\
\hline$h$ & $s_{h}$ & $s^{-}$ & $s^{+}$ & $\underline{s}$ & $\rho_{\mathrm{G}}$ & $s_{h}$ & $s^{-}$ & $s^{+}$ & $\underline{s}$ & $\rho_{\mathrm{G}}$ \\
\hline$h_{\text {ini }}$ & .4060 & .3794 & .5297 & .4546 & .1654 & -.3199 & -.3696 & -.2621 & -.3158 & .1702 \\
\hline $1 / 2 h_{\text {ini }}$ & .4163 & .4061 & .4706 & .4384 & .0736 & -.3203 & -.3438 & -.2982 & -.3210 & .0710 \\
\hline $1 / 4 h_{\text {ini }}$ & .4207 & .4172 & .4423 & .4298 & .0292 & -.3211 & -.3318 & -.3133 & -.3225 & .0286 \\
\hline $1 / 8 h_{\text {ini }}$ & .4224 & .4213 & .4309 & .4261 & .0113 & -.3217 & -.3265 & -.3189 & -.3227 & .0118 \\
\hline
\end{tabular}

Table 2

Example 2: bounds and relative bound gap in a series of uniformly refined $h$-meshes both for $\ell_{0}^{\mathcal{O}}(\boldsymbol{u})$ and $\ell_{1}^{\mathcal{O}}(\boldsymbol{u})$

The values of the bounds for the adaptive procedure with the desired final gap $\Delta_{\text {tol }}=\frac{1}{10} \Delta_{\text {ini }}$ are shown in table 3 . 

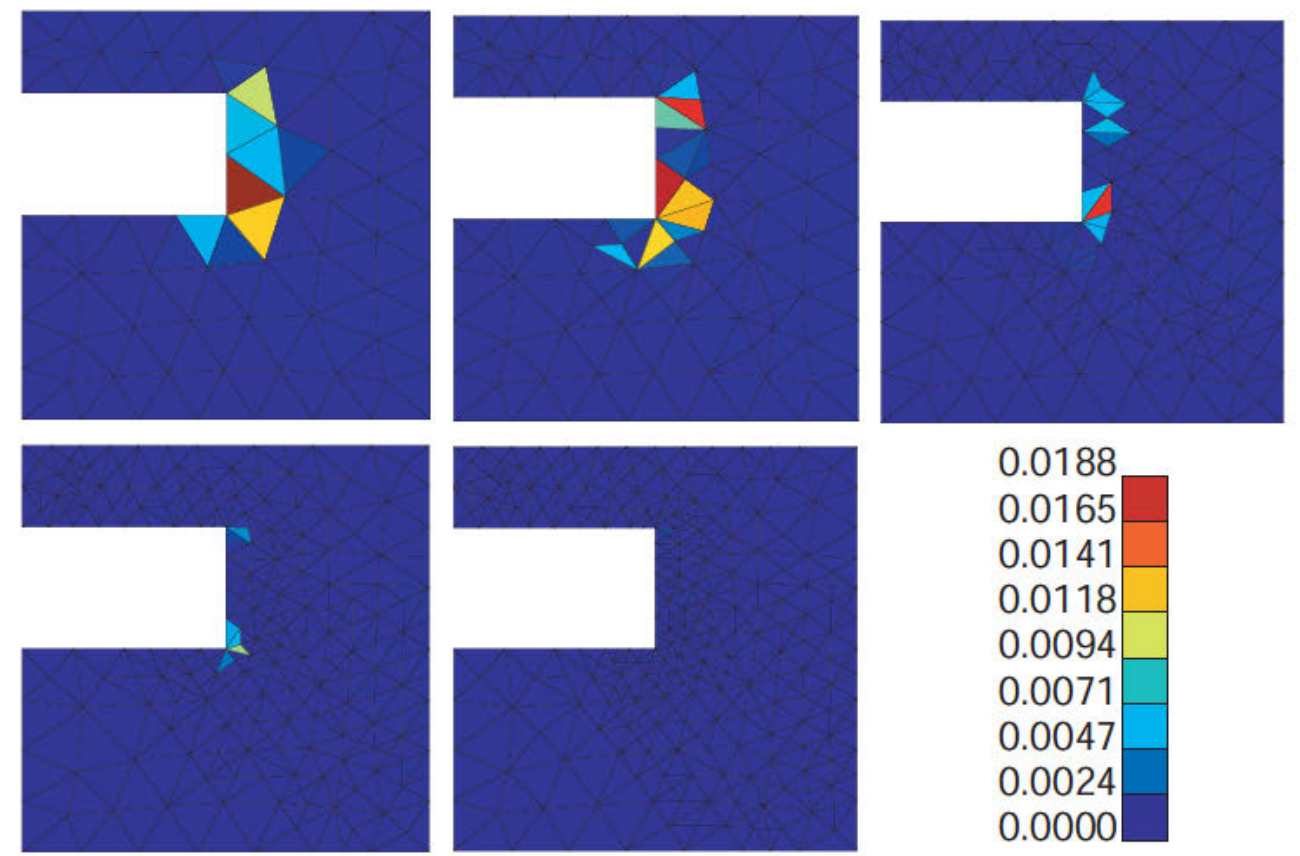

Fig. 6. Example 2: sequence of adapted meshes for the output $\ell_{1}^{\mathcal{O}}(\boldsymbol{u})$ with desired final gap $\Delta_{\text {tol }}=\frac{1}{2} \Delta_{\text {ini }}$ with $\mathrm{n}_{\mathrm{el}}=108,165,280,405$ and 538

\begin{tabular}{|c|c|cc|}
\hline $\mathrm{n}_{\mathrm{e} 1}$ & $\Delta$ & $s_{1}$ & $s_{\mathrm{u}}$ \\
\hline 108 & .10749 & -.36957 & -.26208 \\
222 & .18215 & -.38940 & -.20725 \\
433 & .12171 & -.36880 & -.24709 \\
811 & .07199 & -.35089 & -.27891 \\
1387 & .03755 & -.33750 & -.29995 \\
1966 & .02428 & -.33392 & -.30964 \\
2532 & .01574 & -.32922 & -.31348 \\
3069 & .01172 & -.32826 & -.31654 \\
3564 & .00834 & -.32627 & -.31793 \\
\hline
\end{tabular}

Table 3

Example 2: bounds in a series of adaptively $h$-refined meshes both for $\ell_{1}^{\mathcal{O}}(\boldsymbol{u})$ with desired final gap $\Delta_{\text {tol }}=\frac{1}{10} \Delta_{\text {ini }}$

\section{Conclusions}

We have presented a method for the computation of bounds for linear-functional outputs of weak solutions to the linear elasticity equations. A distinctive feature of this method is that the computed bounds are strict with respect to the output of the exact solution. The numerical experiments presented show that 
the computed bounds are sharp and converge at the same rate as the finite element solution that would be obtained with a comparable amount of work. To our knowledge, this is the only published approach that can certify the certainty of the the computed bounds. We believe this feature is of clear interest in real engineering practice. The method has been presented for the two dimensional elasticity equations, but we expect that the extension to three dimensions will not present any additional difficulties. The major computational cost, in addition to a standard finite element solution, is the computation of an adjoint for each output considered. All other operations are local and result in a low computational overhead. Two limitations in the presented approach are the need for the forcing function to be of piecewise polynomial form, and the requirement for the computational domain to have piecewise straight boundaries. Future work will focus on relaxing these constraints.

\section{Acknowledgments}

Nuria Pares would like to acknowledge the Departament d'Universitats, Recerca i Societat de la Informació of the Generalitat de Catalunya for the support provided to visit MIT. Jaume Peraire would like to acknowledge the generous support provided by the Singapore-MIT Alliance.

\section{Appendix}

\section{A Proof of Theorem 1}

In this appendix we present a constructive proof of Theorem 1 which shows the existence of piecewise polynomial equilibrated stress fields. Towards this end some preliminary notation and results are required (see [4] for details).

Lemma 1 Given a triangle $\mathrm{T}$, consider the following interpolation spaces

$$
\begin{gathered}
P_{q}(\mathrm{~T})=\{\text { polynomial functions of degree less or equal to } q \text { in } \mathrm{T}\}, \\
\boldsymbol{S P}_{q}(\mathrm{~T})=\left\{\text { stress fields with } \sigma_{x x}, \sigma_{x y}, \sigma_{y y} \in P_{q}(\mathrm{~T})\right\}, \\
R_{q}(\partial \mathrm{T})=\left\{\text { polynomial functions of degree less or equal to } q \text { on each } \gamma_{i} \in \partial \mathrm{T}\right\}, \\
\boldsymbol{R}_{q}^{c}(\partial \mathrm{T})=\left\{\boldsymbol{g} \in\left[R_{q}(\partial T)\right]^{2}, \text { s.t. } \exists \boldsymbol{\sigma} \in \boldsymbol{S P}_{q}(\mathrm{~T}), \boldsymbol{\sigma} \cdot \boldsymbol{n}=\boldsymbol{g} \text { on } \partial \mathrm{T}\right\}, \\
\boldsymbol{\Phi}_{q}(\mathrm{~T})=\left\{\boldsymbol{\varsigma}_{q} \in \boldsymbol{S P}_{q}(\mathrm{~T}), \nabla \cdot \boldsymbol{\varsigma}_{q}=0,\left.\boldsymbol{\varsigma}_{q} \cdot n\right|_{\partial T}=0\right\}, \\
\boldsymbol{P}_{\mathrm{sm}}=\{\text { rigid solid body motions in } \mathrm{T}\},
\end{gathered}
$$


and $\boldsymbol{P}_{\mathrm{sm}}^{\perp}$ be the orthogonal complement of $\boldsymbol{P}_{\mathrm{sm}}$ with respect to the standard scalar product in $\left[P_{q-1}(\mathrm{~T})\right]^{2}$, that is, every $\boldsymbol{p} \in\left[P_{q-1}(\mathrm{~T})\right]^{2}$ can be written uniquely as $\boldsymbol{p}=\boldsymbol{p}_{\mathrm{sm}}+\boldsymbol{p}^{\perp}$, with $\boldsymbol{p}_{\mathrm{sm}} \in \boldsymbol{P}_{\mathrm{sm}}$ and $\boldsymbol{p}^{\perp} \in \boldsymbol{P}_{\mathrm{sm}}^{\perp}$. We note that for the case $q=1$, the only member of $\boldsymbol{P}_{\mathrm{sm}}^{\perp}$ is the null function.

Then, for $q \geq 1$, and for any $\boldsymbol{\sigma} \in \boldsymbol{S P}_{q}(\mathrm{~T})$ the following relations imply $\boldsymbol{\sigma}=0$ :

$$
\begin{gathered}
\int_{\partial \mathrm{T}}(\boldsymbol{\sigma} \cdot \boldsymbol{n}) \cdot \boldsymbol{p}_{q} \mathrm{~d} \Gamma=0, \quad \forall \boldsymbol{p}_{q} \in \boldsymbol{R}_{q}^{c}(\partial \mathrm{T}), \\
\int_{\mathrm{T}} \boldsymbol{\sigma}: \boldsymbol{\varepsilon}\left(\boldsymbol{p}_{q-1}\right) \mathrm{d} \Omega=0, \quad \forall \boldsymbol{p}_{q-1} \in \boldsymbol{P}_{\mathrm{sm}}^{\perp} \\
\int_{\mathrm{T}} \boldsymbol{\sigma}: \mathbb{C}^{-1}: \boldsymbol{\varsigma}_{q} \mathrm{~d} \Omega=0, \quad \forall \boldsymbol{\varsigma}_{q} \in \boldsymbol{\Phi}_{q}(\mathrm{~T}),
\end{gathered}
$$

PROOF. First let's check that equations (A.1) and (A.2) imply that $\boldsymbol{\sigma} \in$ $\boldsymbol{\Phi}_{q}(\mathrm{~T})$. Indeed, on one hand, since $\left.\boldsymbol{\sigma} \cdot \boldsymbol{n}\right|_{\partial \mathrm{T}} \in \boldsymbol{R}_{q}^{c}(\partial \mathrm{T})$, from equation (A.1), $\int_{\partial \mathrm{T}}(\boldsymbol{\sigma} \cdot \boldsymbol{n})^{2} \mathrm{~d} \Gamma=0$, which implies that $\boldsymbol{\sigma} \cdot \boldsymbol{n}=0$ in $\partial \mathrm{T}$. On the other hand, the following integration by parts:

$$
\int_{T}(\nabla \cdot \boldsymbol{\sigma}) \cdot(\nabla \cdot \boldsymbol{\sigma}) \mathrm{d} \Omega=\int_{\partial \mathrm{T}}(\boldsymbol{\sigma} \cdot \boldsymbol{n}) \cdot(\nabla \cdot \boldsymbol{\sigma}) \mathrm{d} \Gamma-\int_{\mathrm{T}} \boldsymbol{\sigma}: \boldsymbol{\varepsilon}(\nabla \cdot \boldsymbol{\sigma}) \mathrm{d} \Omega,
$$

plus the fact that $\boldsymbol{\varepsilon}\left(\boldsymbol{p}_{\mathrm{sm}}\right)=0$ for $\boldsymbol{p} \in \boldsymbol{P}_{\mathrm{sm}}$, leads to

$$
\int_{T}(\nabla \cdot \boldsymbol{\sigma}) \cdot(\nabla \cdot \boldsymbol{\sigma}) \mathrm{d} \Omega=\int_{\mathrm{T}} \boldsymbol{\sigma}: \boldsymbol{\varepsilon}\left(\nabla \cdot \boldsymbol{\sigma}-\pi_{\mathrm{sm}}(\nabla \cdot \boldsymbol{\sigma})\right) \mathrm{d} \Omega
$$

where $\pi_{\mathrm{sm}}(\cdot)$ is the projection operator from $\left[P_{q-1}(\mathrm{~T})\right]^{2}$ onto the space $\boldsymbol{P}_{\mathrm{sm}}$. Then, since $\nabla \cdot \boldsymbol{\sigma}-\pi_{\mathrm{sm}}(\nabla \cdot \boldsymbol{\sigma}) \in \boldsymbol{P}_{\mathrm{sm}}^{\perp}$, equation (A.2) implies

$$
\int_{\mathrm{T}}(\nabla \cdot \boldsymbol{\sigma}) \cdot(\nabla \cdot \boldsymbol{\sigma}) \mathrm{d} \Omega=0 \Longrightarrow \nabla \cdot \boldsymbol{\sigma}=0 \text { in } \Omega
$$

which shows that (A.1) and (A.2) imply that $\boldsymbol{\sigma} \in \boldsymbol{\Phi}_{q}(\mathrm{~T})$. Finally, using equation (A.3),

$$
\int_{\mathrm{T}} \boldsymbol{\sigma}: \mathbb{C}^{-1}: \boldsymbol{\sigma} \mathrm{d} \Omega=0 \Longrightarrow \boldsymbol{\sigma}=0
$$

which ends the proof.

Lemma 2 Let $\left\{\boldsymbol{p}_{q}^{i}\right\}_{i=1 \ldots I},\left\{\boldsymbol{p}_{q-1}^{j}\right\}_{j=1 \ldots J}$ and $\left\{\boldsymbol{\varsigma}_{q}^{l}\right\}_{l=1 \ldots L}$ denote the elements of a basis of $\boldsymbol{R}_{q}^{c}(\partial \mathrm{T}), \boldsymbol{P}_{\mathrm{sm}}^{\perp}$ and $\boldsymbol{\Phi}_{q}(\mathrm{~T})$ respectively, where $I, J$ and $L$ simply denote the dimensions of each space. Then, any $\boldsymbol{\sigma} \in \boldsymbol{S P}_{q}(\mathrm{~T})$ is uniquely determined 
by the following degrees of freedom:

$$
\begin{aligned}
& \int_{\partial \mathrm{T}}(\boldsymbol{\sigma} \cdot \boldsymbol{n}) \cdot \boldsymbol{p}_{q}^{i} \mathrm{~d} \Gamma, i \in I, \\
& \int_{\mathrm{T}}^{\boldsymbol{\sigma}}: \boldsymbol{\varepsilon}\left(\boldsymbol{p}_{q-1}^{j}\right) \mathrm{d} \Omega, j \in J, \\
& \int_{\mathrm{T}}^{\boldsymbol{\sigma}}: \mathbb{C}^{-1}: \boldsymbol{\varsigma}_{q}^{l} \mathrm{~d} \Omega, l \in L .
\end{aligned}
$$

PROOF. Lemma 1 states that any stress field $\boldsymbol{\sigma} \in \boldsymbol{S P}_{q}(\mathrm{~T})$ can be described giving the values of the previous degrees of freedom. However, this description it is not necessary unique, that is, different values of the previous degrees of freedom can yield the same stress field. In order to see that this description is unique, it is sufficient to see that the number of degrees of freedom coincides with the dimension of $\boldsymbol{S P}_{q}(\mathrm{~T})$, where $\operatorname{dim}\left(\boldsymbol{S} \boldsymbol{P}_{q}(\mathrm{~T})\right)=\frac{3}{2}(q+1)(q+2)$.

Let's consider first the case $q>1$. It is clear that $\operatorname{dim}\left(\boldsymbol{R}_{q}^{c}(\partial \mathrm{T})\right)=6(q+1)-3$. Now, a basis of $\left[P_{q-1}(\mathrm{~T})\right]^{2}$, determined by $q(q+1)$ elements, defines only $q(q+1)-3$ degrees of freedom of the form $\int_{\mathrm{T}} \boldsymbol{\sigma}: \boldsymbol{\varepsilon}\left(\boldsymbol{p}_{q-1}\right) \mathrm{d} \Omega$, since $\boldsymbol{\varepsilon}\left(\boldsymbol{t}_{x}\right)=$ $\boldsymbol{\varepsilon}\left(\boldsymbol{t}_{y}\right)=\boldsymbol{\varepsilon}(\boldsymbol{r})=0$, for $\boldsymbol{t}_{x}, \boldsymbol{t}_{y}$ and $\boldsymbol{r}$ the three rigid solid body motions, that is, $\operatorname{dim}\left(\boldsymbol{P}_{\mathrm{sm}}^{\perp}\right)=q(q+1)-3$.

Then, the only remaining part is to determine the dimension of $\boldsymbol{\Phi}_{q}(\mathrm{~T})$. Any $\boldsymbol{\varsigma} \in \boldsymbol{\Phi}_{q}(\mathrm{~T})$ can be rewritten as $\varsigma_{x x}=\partial^{2} b / \partial^{2} y, \varsigma_{y y}=\partial^{2} b / \partial x^{2}, \varsigma_{x y}=\varsigma_{y x}=$ $-\partial^{2} b / \partial x \partial y$, where $b \in b_{T}^{2} P_{q-4}(\mathrm{~T})$ for $b_{T}$ the cubic bubble function on $\mathrm{T}$ vanishing on $\partial \mathrm{T}$ and achieving a maximum value of unity on $\mathrm{T}$, see [2]. Therefore, $\operatorname{dim}\left(\boldsymbol{\Phi}_{q}(\mathrm{~T})\right)=\operatorname{dim}\left(P_{q-4}(\mathrm{~T})\right)=\frac{1}{2}(q-2)(q-3)$.

Finally, it is trivial to check that:

$$
\underbrace{\frac{3}{2}(q+1)(q+2)}_{\operatorname{dim}\left(\boldsymbol{S P}_{q}(\mathrm{~T})\right)}=\underbrace{6(q+1)-3}_{\operatorname{dim}\left(\boldsymbol{R}_{q}^{c}(\partial \mathrm{T})\right)}+\underbrace{q(q+1)-3}_{\operatorname{dim}\left(\boldsymbol{P}_{\mathrm{sm}}^{\perp}\right)}+\underbrace{\frac{1}{2}(q-2)(q-3)}_{\operatorname{dim}\left(\boldsymbol{\Phi}_{q}(\mathrm{~T})\right)} .
$$

For the particular case $q=1,\left[P_{0}(\mathrm{~T})\right]^{2}=<\boldsymbol{t}_{x}, \boldsymbol{t}_{y}>$, and equations $\int_{\mathrm{T}} \boldsymbol{\sigma}$ : $\varepsilon\left(\boldsymbol{p}_{0}\right) \mathrm{d} \Omega=0$ do not characterize any degree of freedom. Moreover, in this case $\operatorname{dim}\left(\Phi_{1}(\mathrm{~T})\right)=0$. Then the 9 boundary degrees of freedom $R_{1}^{c}(\partial \mathrm{T})$ determine uniquely the linear stress field in the triangle.

We can now proceed directly with the proof of Theorem 1 . We will assume that the initial element $\Omega_{k}$ is a triangle, but the strategy can be easily extended to quadrilateral elements. In order to find $\boldsymbol{\sigma}_{k}$, we follow [10] and divide the initial triangle into three new triangles, $\Omega_{k}=\mathrm{T}_{1} \cup \mathrm{T}_{2} \cup \mathrm{T}_{3}$ by adding a point in the triangle centroid, as indicated in figure (A.1). 


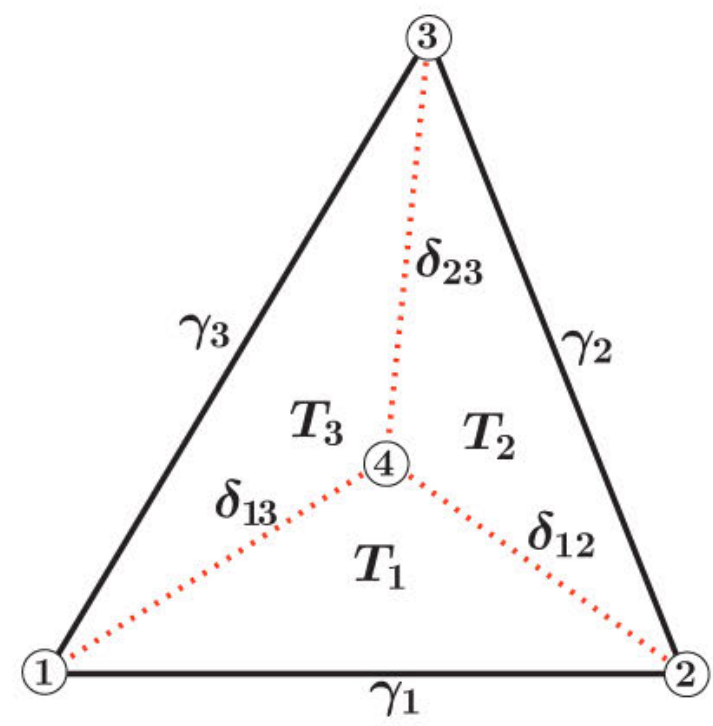

Fig. A.1. Local subdivision of an element $\Omega_{k}$ into the triangles $\mathrm{T}_{1}, \mathrm{~T}_{2}$ and $\mathrm{T}_{3}$

Let, $\boldsymbol{\sigma}_{\boldsymbol{\lambda}}$ be a stress field in $\Omega_{k}$, where $\left.\boldsymbol{\sigma}_{\boldsymbol{\lambda}}\right|_{\mathrm{T}_{i}} \in \boldsymbol{S P}_{q}\left(\mathrm{~T}_{i}\right), i=1,2,3$, that is, $\sigma_{\boldsymbol{\lambda}}$ is a polynomial stress field of degree $q$ in each subtriangle, such that, $\left.\boldsymbol{\sigma}_{\boldsymbol{\lambda}}\right|_{\partial \mathrm{T}}=\tau_{k} \boldsymbol{\lambda}_{h}$, and with continuous normal tractions at the internal edges of the partition $\left(\delta_{i j}=\mathrm{T}_{i} \cap \mathrm{T}_{j}, i, j=1 \ldots 3\right)$.

Then, setting $\sigma_{k}=\sigma_{\boldsymbol{\lambda}}-\boldsymbol{\sigma}\left(\left.\boldsymbol{u}^{*}\right|_{\mathrm{T}}\right)+\boldsymbol{\sigma}_{0}$, the initial problem reduces to finding a piecewise polynomial stress field $\sigma_{0}$ verifying:

$$
\int_{\Omega_{k}} \sigma_{0}: \varepsilon(v) \mathrm{d} \Omega=\int_{\Omega_{k}}\left(f^{*}+\nabla \cdot \sigma_{\boldsymbol{\lambda}}\right) \cdot \boldsymbol{v} \mathrm{d} \Omega, \quad \forall \boldsymbol{v} \in \mathcal{V}_{k}
$$

Let $\sigma_{0}$ be a piecewise polynomial stress field, where in each triangle $\mathrm{T}_{i}$, the stress field $\sigma_{0}^{i}=\left.\sigma_{0}\right|_{\mathrm{T}_{i}}$ is assumed to be polynomial of degree $q$ in each component, that is, $\boldsymbol{\sigma}_{0}^{i} \in \boldsymbol{S P}_{q}\left(\mathrm{~T}_{i}\right)$.

Then, $\sigma_{0}$ is uniquely determined by the degrees of freedom characterized in Lemma 2. First, there are the $18(q+1)-9$ degrees of freedom determining the value of $\sigma_{0}$ at the edges of $\Omega_{k}$ and at the internal edges, namely

$$
\int_{\partial \mathrm{T}_{i}}\left(\boldsymbol{\sigma}_{0}^{i} \cdot \boldsymbol{n}\right) \cdot \boldsymbol{p} \mathrm{d} \Gamma, \quad \forall \boldsymbol{p} \in \boldsymbol{R}_{q}^{c}\left(\partial \mathrm{T}_{i}\right), i=1 \ldots 3 .
$$

Second, we have the degrees of freedom related to the divergence of $\sigma_{0}$, that is

$$
\int_{\mathrm{T}_{i}} \boldsymbol{\sigma}_{0}^{i}: \boldsymbol{\varepsilon}(\boldsymbol{p}) \mathrm{d} \Omega, \quad \forall \boldsymbol{p} \in \boldsymbol{P}_{\mathrm{sm}}\left(\mathrm{T}_{i}\right)^{\perp}, i=1 \ldots 3 .
$$

And finally, the degrees of freedom associated to $\Phi_{q}\left(\mathrm{~T}_{i}\right), i=1,2,3$, which can be set arbitrarily.

The proof ends with the construction of $\sigma_{0}$ that verifies (A.4). This construction also follows the classification of Lemma 2: (i) the boundary degrees of 
freedom, (ii) the divergence ones and (iii) the related to $\boldsymbol{\Phi}_{q}\left(\mathrm{~T}_{i}\right)$, which are not detailed because they are arbitrary.

(i) The $18(q+1)-9$ boundary degrees of freedom are determined in two steps. First, the $12(q+1)$ constraints to enforce compatibility are imposed

$$
\int_{\gamma_{i}}\left(\boldsymbol{\sigma}_{0}^{i} \cdot \boldsymbol{n}\right) \cdot \boldsymbol{p} \mathrm{d} \Gamma=0, \quad \forall \boldsymbol{p} \in\left[R_{q}\left(\gamma_{i}\right)\right]^{2}, i=1 \ldots 3,
$$

where $\gamma_{i}=\partial \mathrm{T}_{i} \cap \partial \mathrm{T}$, and

$$
\int_{\delta_{i j}}\left(\boldsymbol{\sigma}_{0}^{i} \cdot \boldsymbol{n}\right) \cdot \boldsymbol{p} \mathrm{d} \Gamma=\int_{\delta_{i j}}\left(\boldsymbol{\sigma}_{0}^{j} \cdot \boldsymbol{n}\right) \cdot \boldsymbol{p} \mathrm{d} \Gamma, \quad \forall \boldsymbol{p} \in\left[R_{q}\left(\delta_{i j}\right)\right]^{2}, i, j=1 \ldots 3, i<j .
$$

Then, the $6 q-3$ remainder degrees of freedom are used to impose the following additional constraints,

$$
\int_{\partial \mathrm{T}_{i}}\left(\boldsymbol{\sigma}_{0}^{i} \cdot \boldsymbol{n}\right) \cdot \boldsymbol{v} \mathrm{d} \Omega=-\int_{\mathrm{T}_{i}}\left(\boldsymbol{f}^{*}+\nabla \cdot \boldsymbol{\sigma}_{\boldsymbol{\lambda}}\right) \cdot \boldsymbol{v} \mathrm{d} \Omega, \quad \forall \boldsymbol{v} \in \boldsymbol{P}_{\mathrm{sm}}\left(\mathrm{T}_{i}\right), i=1 \ldots 3 .
$$

It is important to note that since $\boldsymbol{\lambda}_{h}$ and $\boldsymbol{f}^{*}$ verify equation (22), some of the previous equations are redundant. For $q>1$, equation (A.7) represents 9 constraints but only 6 degrees of freedom are required to impose them because equation (22) is scalar and $\operatorname{dim}\left(\boldsymbol{P}_{\mathrm{sm}}\right)=3$. In the case $q=1$, equation (A.7) represents 6 constraints but only 3 of them are independent for the same reason. Note that for $q=1$, the boundary degrees of freedom are uniquely determined, while for $q>1$, there are $6 q-9$ degrees of freedom left associated to the internal boundaries which can be set arbitrarily.

(ii) Once the boundary degrees of freedom are fixed, we impose those related to the divergence of $\boldsymbol{\sigma}_{0}$, namely

$$
\int_{\mathrm{T}_{i}} \boldsymbol{\sigma}_{0}^{i}: \boldsymbol{\varepsilon}(\boldsymbol{p}) \mathrm{d} \Omega=\int_{\mathrm{T}_{i}}\left(\boldsymbol{f}^{*}+\nabla \cdot \boldsymbol{\sigma}_{\boldsymbol{\lambda}}\right) \cdot \boldsymbol{p} \mathrm{d} \Omega+\int_{\partial \mathrm{T}_{i}}\left(\boldsymbol{\sigma}_{0}^{i} \cdot \boldsymbol{n}\right) \cdot \boldsymbol{p} \mathrm{d} \Gamma,
$$

for all $\boldsymbol{p} \in \boldsymbol{P}_{\mathrm{sm}}\left(\mathrm{T}_{i}\right)^{\perp}, i=1 \ldots 3$.

Once (A.7) and in (A.8) have been imposed, $\boldsymbol{\sigma}_{0}$ verifies

$$
\int_{\mathrm{T}_{i}} \boldsymbol{\sigma}_{0}^{i}: \boldsymbol{\varepsilon}(\boldsymbol{p}) \mathrm{d} \Omega=\int_{\mathrm{T}_{i}}\left(\boldsymbol{f}^{*}+\nabla \cdot \boldsymbol{\sigma}_{\boldsymbol{\lambda}}\right) \cdot \boldsymbol{p} \mathrm{d} \Omega+\int_{\partial \mathrm{T}_{i}}\left(\boldsymbol{\sigma}_{0}^{i} \cdot \boldsymbol{n}\right) \cdot \boldsymbol{p} \mathrm{d} \Gamma,
$$

for all $\boldsymbol{p} \in\left[P_{q-1}\left(\mathrm{~T}_{i}\right)\right]^{2}, i=1 \ldots 3$.

To conclude the proof it only remains to show that the stress field $\boldsymbol{\sigma}_{0}$ indeed verifies (A.4). On one hand, a simple integration by parts shows that equation (A.9) is equivalent to

$$
\int_{\mathrm{T}_{i}}\left(\nabla \cdot \boldsymbol{\sigma}_{0}^{i}-\left(\boldsymbol{f}^{*}+\nabla \cdot \boldsymbol{\sigma}_{\boldsymbol{\lambda}}\right)\right) \cdot \boldsymbol{p} \mathrm{d} \Omega=0, \forall \boldsymbol{p} \in\left[P_{q-1}\left(\mathrm{~T}_{i}\right)\right]^{2}, i=1 \ldots 3
$$


Since $\nabla \cdot \boldsymbol{\sigma}_{0}^{i}, \boldsymbol{f}^{*}$ and $\nabla \cdot \boldsymbol{\sigma}_{\boldsymbol{\lambda}} \in\left[P_{q-1}\left(\mathrm{~T}_{i}\right)\right]^{2}$, we have $\nabla \cdot \boldsymbol{\sigma}_{0}^{i}=\boldsymbol{f}^{*}+\nabla \cdot \boldsymbol{\sigma}_{\boldsymbol{\lambda}}$, and thus equations (A.9) and (A.10) hold not only for $\boldsymbol{p} \in\left[P_{q-1}\left(\mathrm{~T}_{i}\right)\right]^{2}$ but for $\boldsymbol{p} \in\left[\mathcal{H}^{1}\left(\mathrm{~T}_{i}\right)\right]^{2}$. One the other hand, using a similar reasoning, we have that $\boldsymbol{\sigma}_{0}^{i} \in \boldsymbol{S P}_{q}\left(\mathrm{~T}_{i}\right)$ and equations (A.5), (A.6) give $\left.\boldsymbol{\sigma}_{0}^{i} \cdot \boldsymbol{n}\right|_{\gamma_{i}}=0, i=1 \ldots 3$ and $\left.\left(\boldsymbol{\sigma}_{0}^{i}-\boldsymbol{\sigma}_{0}^{j}\right) \cdot \boldsymbol{n}\right|_{\delta_{i j}}=0, i, j=1 \ldots 3, i<j$. Thus,

$$
\int_{\gamma_{i}}\left(\boldsymbol{\sigma}_{0}^{i} \cdot \boldsymbol{n}\right) \cdot \boldsymbol{p} \mathrm{d} \Gamma=0, \forall \boldsymbol{p} \in\left[R_{q}\left(\gamma_{i}\right)\right]^{2}, i=1 \ldots 3,
$$

where $\gamma_{i}=\partial \mathrm{T}_{i} \cap \partial \mathrm{T}$, and

$$
\int_{\delta_{i j}}\left(\boldsymbol{\sigma}_{0}^{i} \cdot \boldsymbol{n}\right) \cdot \boldsymbol{p} \mathrm{d} \Gamma=\int_{\delta_{i j}}\left(\boldsymbol{\sigma}_{0}^{j} \cdot \boldsymbol{n}\right) \cdot \boldsymbol{p} \mathrm{d} \Gamma, \forall \boldsymbol{p} \in\left[R_{q}\left(\delta_{i j}\right)\right]^{2}, i, j=1 \ldots 3, i<j .
$$

hold, not only for $\boldsymbol{p} \in\left[\boldsymbol{R}_{q}^{c}\left(\gamma_{i}\right)\right]^{2}$ and $\boldsymbol{p} \in\left[\boldsymbol{R}_{q}^{c}\left(\delta_{i j}\right)\right]^{2}$, but for $\boldsymbol{p} \in\left[\mathcal{H}^{\frac{1}{2}}\left(\gamma_{i}\right)\right]^{2}$ and $\boldsymbol{p} \in\left[\mathcal{H}^{\frac{1}{2}}\left(\delta_{i j}\right)\right]^{2}$ respectively. Finally, for any $\boldsymbol{v} \in \mathcal{V}_{k}=\left[\mathcal{H}^{1}(\mathrm{~T})\right]^{2}$ using the infinite dimensional versions of equations (A.5), (A.6) and (A.9), and the fact that $\boldsymbol{v}$ is continuous on $\delta_{i j}$,

$$
\begin{aligned}
\int_{\mathrm{T}} \boldsymbol{\sigma}_{0}: \boldsymbol{\varepsilon}(\boldsymbol{v}) \mathrm{d} \Omega & =\sum_{i=1}^{3} \int_{\mathrm{T}_{i}} \boldsymbol{\sigma}_{0}^{i}: \boldsymbol{\varepsilon}(\boldsymbol{v}) \mathrm{d} \Omega= \\
& =\int_{\mathrm{T}}\left(\boldsymbol{f}^{*}+\nabla \cdot \boldsymbol{\sigma}_{\boldsymbol{\lambda}}\right) \cdot \boldsymbol{v} \mathrm{d} \Omega+\underbrace{\sum_{i=1}^{3} \int_{\partial \mathrm{T}_{i}}\left(\boldsymbol{\sigma}_{0}^{i} \cdot \boldsymbol{n}\right) \cdot \boldsymbol{v} \mathrm{d} \Gamma}_{0} \\
& =\int_{\mathrm{T}}\left(\boldsymbol{f}^{*}+\nabla \cdot \boldsymbol{\sigma}_{\boldsymbol{\lambda}}\right) \cdot \boldsymbol{v} \mathrm{d} \Omega
\end{aligned}
$$

ending the proof.

\section{References}

[1] Ainsworth M., Oden J.T., A posteriori error estimation in finite element analysis, Comp. Meths. Appl. Mech. Engr., 142:1-88, 1997.

[2] Arnold D.N., Winther R., Mixed finite element for elasticity. Numerische Mathematik 2002;92:401-419.

[3] Bank R.E., Weiser A., Some a posteriori error indicators for elliptic partial differential equations, Mat. Comp., 44:170, 1895, pp. 283-301.

[4] Brezzi F., Fortin M., Mixed and Hybrid Finite Element Methods, Springer Series in Computational mathematics, Springer-Verlag, 1991.

[5] Destuynder P., Métivet B., Explicit error bounds in a conforming finite element method. Mathematics of Computation 1999; 68:1379-1396. 
[6] Díez P., Parés N., Huerta A., Recovering lower bounds of the error postprocessing implicit residual a posteriori error estimates. International Journal for Numerical Methods in Engineering 2003;56:1465-1488.

[7] Fraeijs de Veubeke J. Displacement and equilibrium models in the finite element method. B. M. Fraeijs de Veubeke Memorial Volume of Selected Papers 1980; Int. J. Numer. Meth. Engr.. Classical Reprint Series 2001; 52:287-342.

[8] Kelly D. The self-equilibration of residuals and complementary a posteriori error estimates in the finite element method. Int. J. Numer. Meth. Engr. 1984; 20:1491-1506.

[9] Ladevèze P., Leguillon D., Error estimate procedure in the finite element method and applications. . SIAM Journal of Numerical Analysis 1983; 20:485-509.

[10] Ladevèze P., Pelle J.P., Rougeot P., Error estimation and mesh optimization for classical finite elements. Engrg. Comput. 1991; 8:69-80.

[11] Paraschivoiu M., Patera A.T., A hierarchical duality approach to bounds for the outputs of partial differential equations, Comp. Meths. Appl. Mech. Engr. 1998, 158(3-4):389-407.

[12] Paraschivoiu M., Peraire J., Patera A.T. A posteriori finite element bounds for linear functional outputs of elliptic partial differential equations. Comp. Meths. Appl. Mech. Engr. 1997; 1580:289-312.

[13] Peraire J., Patera A.T. Bounds for linear-functional outputs of coercive partial differential equations: Local indicators and adaptive refinement, in Proceedings of the Workshop On New Advances in Adaptive Computational Methods in Mechanics, P. Ladeveze and J. Oden, eds., Cachan, September 17-19, 1997, Elsevier.

[14] Prudhomme S., Oden J.T. On Goal-Oriented Error Estimation for Elliptic Problems: Application to the Control of Pointwise Errors. Comp. Meths. Appl. Mech. Engr. 1999; 176:313-331.

[15] Prudhomme S., Oden J.T., Westermann T., Bass J., Botkin M.E., Practical methods for a posteriori error estimation in engineering applications. International Journal for Numerical Methods in Engineering 2003;56:1193-1224.

[16] Rannacher R., Suttmeier F.-T., A feed-back approach to error control in finite elemetn methods: application to linear elasticity, Computational Mechnics, 19:434-446, 1997.

[17] Roca X., Sarrate J., Huerta A., Una librería orientada al objeto para el refinamiento de triangulos y tetraedros. Aplicaciones al cálculo adaptado. In proceedings of Métodos Computacionais em Engenharia. Lisbon 2004.

[18] Sauer-Budge A.M, Bonet J., Huerta A., Peraire J. Computing bounds for linear functionals of exact weak solutions to Poisson's equation. Accepted for publication in SIAM Journal on Numerical Analysis 2003. 
[19] Sauer-Budge A.M., Peraire J., Computing bounds for linear functionals of exact weak solutions to the advection-diffusion-reaction equation. Accepted for Publication in SIAM Journal on Scientific Computing 2003.

[20] Strouboulis T., Babuska I., Guraranteed computable bounds for the exact error in the finite element solution - Part II: bounds for the energy norm of the error in two dimensions, Int. J. Numer. Meth. Engnr. 47, 427-475, 2000. 